OPEN ACCESS

Edited by:

Karoline Faust,

KU Leuven, Belgium

Reviewed by:

Abhinav Achreja,

University of Michigan, United States

Adam Martiny,

University of California, Irvine,

United States

Steffen Waldherr,

KU Leuven, Belgium

*Correspondence:

Daniel Segrè

dsegre@bu.edu

${ }^{\dagger}$ These authors have contributed equally to this work

Specialty section:

This article was submitted to

Systems Biology,

a section of the journal

Frontiers in Genetics

Received: 22 July 2020 Accepted: 14 January 2021

Published: 09 February 2021

Citation:

Ofaim S, Sulheim S, Almaas E, Sher D and Segrè D (2021) Dynamic

Allocation of Carbon Storage and Nutrient-Dependent Exudation in a Revised Genome-Scale Model

of Prochlorococcus.

Front. Genet. 12:586293.

doi: 10.3389/fgene.2021.586293

\section{Dynamic Allocation of Carbon Storage and Nutrient-Dependent Exudation in a Revised Genome-Scale Model of Prochlorococcus}

\author{
Shany Ofaim ${ }^{1,2 \dagger}$, Snorre Sulheim ${ }^{1,3,4 \dagger}$, Eivind Almaas ${ }^{3,5}$, Daniel Sher ${ }^{2}$ and \\ Daniel Segrè $1,6,7,8 *$
}

'Bioinformatics Program and Biological Design Center, Boston University, Boston, MA, United States, ${ }^{2}$ Department of Marine Biology, University of Haifa, Haifa, Israel, ${ }^{3}$ Department of Biotechnology and Food Science, NTNU - Norwegian University of Science and Technology, Trondheim, Norway, ${ }^{4}$ Department of Biotechnology and Nanomedicine, SINTEF Industry, Trondheim, Norway, ${ }^{5}$ K. G. Jebsen Center for Genetic Epidemiology, NTNU - Norwegian University of Science and Technology, Trondheim, Norway, ${ }^{6}$ Department of Biomedical Engineering, Boston University, Boston, MA, United States, ${ }^{7}$ Department of Physics, Boston University, Boston, MA, United States, ${ }^{8}$ Department of Biology, Boston University, Boston, MA, United States

Microbial life in the oceans impacts the entire marine ecosystem, global biogeochemistry and climate. The marine cyanobacterium Prochlorococcus, an abundant component of this ecosystem, releases a significant fraction of the carbon fixed through photosynthesis, but the amount, timing and molecular composition of released carbon are still poorly understood. These depend on several factors, including nutrient availability, light intensity and glycogen storage. Here we combine multiple computational approaches to provide insight into carbon storage and exudation in Prochlorococcus. First, with the aid of a new algorithm for recursive filling of metabolic gaps (ReFill), and through substantial manual curation, we extended an existing genome-scale metabolic model of Prochlorococcus MED4. In this revised model (iSO595), we decoupled glycogen biosynthesis/degradation from growth, thus enabling dynamic allocation of carbon storage. In contrast to standard implementations of flux balance modeling, we made use of forced influx of carbon and light into the cell, to recapitulate overflow metabolism due to the decoupling of photosynthesis and carbon fixation from growth during nutrient limitation. By using random sampling in the ensuing flux space, we found that storage of glycogen or exudation of organic acids are favored when the growth is nitrogen limited, while exudation of amino acids becomes more likely when phosphate is the limiting resource. We next used COMETS to simulate day-night cycles and found that the model displays dynamic glycogen allocation and exudation of organic acids. The switch from photosynthesis and glycogen storage to glycogen depletion is associated with a redistribution of fluxes from the Entner-Doudoroff to 
the Pentose Phosphate pathway. Finally, we show that specific gene knockouts in iSO595 exhibit dynamic anomalies compatible with experimental observations, further demonstrating the value of this model as a tool to probe the metabolic dynamic of Prochlorococcus.

Keywords: constraint-based reconstruction and analysis (COBRA), flux balance analysis (FBA), computation of microbial ecosystems in time and space (COMETS), cyanobacteria, exudation, gap-filling algorithm, photosynthesis

\section{INTRODUCTION}

Marine phytoplankton perform about one-half of the photosynthesis on Earth (Field et al., 1998). Prochlorococcus is one of the most abundant phytoplankton clades in the world's oceans and is estimated to produce about $4 \mathrm{Gt}$ of organic carbon annually (Flombaum et al., 2013). As such, these clades play a key role in a variety of ecosystems (Partensky and Garczarek, 2010; Biller et al., 2015). Recent evolutionary studies suggested several evolved metabolic innovations contributing to high picocyanobacterial abundance in the harsh oligotrophic ocean waters, usually limited by several nutrients such as nitrogen, phosphorus, and iron. These innovations include a proteome that contains less nitrogen rich amino acids (Gilbert and Fagan, 2011), membranes that contain glyco- and sulfolipids rather than phospholipids (Van Mooy et al., 2006) and streamlining of the genome associated with outsourcing of important cellular functions to co-occurring organisms (Holtzendorff et al., 2008; Partensky and Garczarek, 2010; Morris et al., 2012; Ma L. et al., 2018; Braakman, 2019).

Another innovation employed by these organisms is an increased metabolic rate that in turn manifest in the exudation of organic compounds (Fogg et al., 1965; Mague et al., 1980; López-Sandoval et al., 2013; Braakman et al., 2017; Braakman, 2019; Moran and Durham, 2019). Typically, 2-25\% of the carbon fixed by photosynthesis is released by exudation from the cell, although values as high as $90 \%$ have been reported (Bertilsson et al., 2005; López-Sandoval et al., 2013; Rothrosenberg et al., 2019; Szul et al., 2019). This exudation, combined with cell death, lytic viral infections, and grazing debris made by predators ("sloppy feeding"), makes dissolved organic matter of phytoplankton origin omnipresent in natural waters (Thornton, 2014). However, it is currently impossible to provide a universal chemical description of dissolved organic matter (Kujawinski, 2011; Arrieta et al., 2015; Moran et al., 2016), partly because the exuded organic compounds differ between strains and environmental conditions (Becker et al., 2014; Ma X. et al., 2018). Nevertheless, in general, phytoplankton exudate includes a small proportion of low-molecular weight compounds, such as organic acids, carbohydrates, and amino acids (Bertilsson et al., 2005), as well as a larger proportion of complex, high-molecular weight compounds (Kujawinski, 2011). Another strategy employed by these bacteria to manage their carbon budget is the internal storage of carbon in polymeric form, specifically, glycogen (Zinser et al., 2009; Reimers et al., 2017; Luan et al., 2019). The extent to which Prochlorococcus, in particular, also stores glycogen has recently been measured, showing increased glycogen pools (up to $40 \mathrm{fg} \mathrm{cell}^{-1}$ ) in nitrogenlimited conditions compared to nitrogen-replete (Szul et al., 2019). Glycogen accumulates in the bacterial cell during the light hours and was recently suggested to have two primary roles; as energy storage in preparation for darkness and as a regulation strategy to manage high-light photosynthesis products (Welkie et al., 2019). The allocation of glycogen is suggested to be tightly associated with the overflow metabolism hypothesis and also known to be widely affected by nutrient limitations (Damrow et al., 2016; Cano et al., 2018; Forchhammer and Schwarz, 2019; Szul et al., 2019). Importantly, the carbon fixed and released by phytoplankton is then used by heterotrophic organisms as a source of energy, whereas the heterotrophic bacteria may recycle nutrient elements and support the growth of phytoplankton in other ways, as suggested by the Black Queen Hypothesis (Amin et al., 2012; Morris et al., 2012; Moran et al., 2016; Cirri and Pohnert, 2019; Moran and Durham, 2019). Thus, carbon fixation, storage and release are tightly intertwined with microbial interactions and microbial ecosystem dynamics.

Quantitative models at various scales have provided critical insights into how ocean microbial ecosystems function, and how they are related to broader biogeochemical cycles (Deutsch et al., 2007; Follows et al., 2007; Arteaga et al., 2016; Coles et al., 2017; Foster et al., 2018; Moradi et al., 2018; Nicholson et al., 2018; Braakman, 2019; Oschlies et al., 2019; Ward et al., 2019). Most of these models represent organisms in terms of simplified stoichiometric reactions converting elements into biomass, thus making it possible to incorporate biological processes into dynamic-coupled Earth System models (Follows et al., 2007; Reid, 2012). The exponential increase in genomic information on marine organisms provides an opportunity to seek methods to link such detailed genome-scale information to biochemical flows (Coles et al., 2017). In recent years, genome-scale metabolic models (GEMs), combined with linear programming, have made it possible to produce testable predictions of metabolic phenotypes of individual organisms or microbial communities (Gu et al., 2019). This computational framework is based on the identification of individual enzymes and transporters in an organism's genome, and on simplifying assumptions that bypass the need for kinetic parameters (Maarleveld et al., 2013; O’Brien et al., 2015; Casey et al., 2016; Kim et al., 2016; Reimers et al., 2017). While genome-scale modeling has proven to be a powerful approach in cyanobacterial model organisms such as Synechocystis sp. PCC 6803, Synechococcus elongatus PCC 7942 and Prochlorococcus MED4 (Kettler et al., 2007; Knoop et al., 2013; Broddrick et al., 2016; Casey et al., 2016; Yoshikawa et al., 2017), the exudation of organic compounds in 
phototrophic organisms has not been studied in detail through Flux Balance Analysis (FBA) or similar methods (Varma and Palsson, 1994; Orth et al., 2010). On the other hand, several examples exist of FBA-based predictions of exudation-mediated interactions between different species, including those generated using the Computational of Microbial Ecosystems in Time and Space (COMETS) platform (Harcombe et al., 2014). In fact, FBA calculations also suggest that "costless" secretions (i.e., secretions that do not induce a fitness cost) might be quite common, and can support the growth of co-occurring organisms (Pacheco et al., 2018).

Experimental evidence and theoretical considerations indicate that Prochlorococcus exudes different metabolites in a way that strongly depends on environmental conditions (Dubinsky and Berman-Frank, 2001; Szul et al., 2019) as well as on the strain's genetic makeup (Becker et al., 2014; Roth-rosenberg et al., 2019). While GEMs can be used to predict these fluxes, they require modifications to deal with processes not usually considered in FBA, including: (a) the special nature of photon fluxes [which, unlike molecular fluxes, cannot easily be "shut off" at short time scales (Dubinsky and Berman-Frank, 2001)]; (b) the buffering role of intracellular storage molecules such as glycogen. The primary focus of this study is to obtain better knowledge of the potential metabolic effect of a combination of key nutrients (carbon, nitrogen, phosphorous, and light) and carbon fixation rate on the allocation (including storage and exudation) of carbon in Prochlorococcus using a revised genome-scale metabolic model (Figure 1). We start by describing model revisions and updates to capture the current, most complete metabolic knowledge available for Prochlorococcus. Next, we use a variety of FBA approaches to uncover the potential relationships between a set of key nutrients, carbon storage and exudates in static and dynamic (time dependent) settings. The implementation and use of these approaches improve our understanding of the intricate metabolic workings of Prochlorococcus and provide insights on its storage and exudation trends under different environmental conditions.

\section{MATERIALS AND METHODS}

\section{Model Update and Curation}

The iJC568 genome-scale reconstruction of Prochlorococcus marinus subsp. pastoris str. CMP1986 (referred to throughout the manuscript as MED4) as described by Casey et al. (2016), was used as the starting point for model enhancement. The update process started with an in-depth study of the reconstructed network and available knowledge not previously incorporated into the model of the organism. During this process, we ended up implementing the following specific steps of curation and update: (i) A key modification to the model was the decoupling between the glycogen storage flux and the biomass production. In standard stoichiometric reconstructions for FBA modeling (Thiele et al., 2011; Nogales et al., 2012; Feist et al., 2014; Broddrick et al., 2016; Monk et al., 2017; Kavvas et al., 2018), glycogen is listed as one of the biomass components, thus accounting for the carbon flux into storage. However, given the fixed stoichiometry of biomass composition, this classical implementation cannot account for the time-dependent storage and re-utilization of glycogen observed in picocyanobacteria. We thus removed the glycogen from the biomass function and streamlined the existing glycogen granule representation to a direct link between ADP-Glucose to the production of glycogen (Figure 1B). (ii) In addition to targeted refinement of selected reactions, we used the KEGG database (Kanehisa and Goto, 2000) to perform an extensive search for previously known but missing metabolic reaction annotations. Indeed, we found 354 reactions that could be potentially added to the existing network. To incorporate this knowledge, we developed a semi-automated algorithm (ReFill, described below). (iii) We coupled the implementation of the algorithm with several steps of manual curation. These included the addition of transports, such as that of hydrogen peroxide and ethanol, known to diffuse across the cell membrane (Seaver and Imlay, 2001; Noreña-Caro and Benton, 2018), and the addition of the complete Entner-Doudoroff pathway, that has recently been discovered in cyanobacteria (Chen et al., 2016). Additionally, we performed a BLAST search (Supplementary Material 1) (Altschul et al., 1990) from which we identified 6PG-dehydratase (EC: 4.2.1.12) encoded by PMM0774, thereby completing this pathway in the model reconstruction. (iv) The revised model was checked for redox and elemental balance. Since the biomass function was based on experimental data (Casey et al., 2016), it was not updated. In line with best practices, a memote quality assessment (Supplementary Material 2) (Lieven et al., 2020), as well as model files and a detailed changelog, are provided at https://github.com/segrelab/Prochlorococcus_ Model. All reactions added to iJC658 to form iSO595 are found in Supplementary Table $\mathbf{1}$ and modified reactions are found in Supplementary Table 2 .

\section{ReFill Algorithm}

Following an extensive search of literature and the KEGG (Kanehisa and Goto, 2000), TransportDB (Elbourne et al., 2017) and Metabolights (Haug et al., 2013) databases, we found a large number of new or previously known but missing reaction, transporter, and metabolite annotations. Adding large amounts of data to an existing network might create new gaps and may give rise to new blocked reactions and orphan metabolites that in general reduce model quality and can convolute later curation efforts, quality control or assessment of model predictions. To add this knowledge to the network in a controlled approach, we developed the semi-automated recursive algorithm ReFill (Recursive Filler of metabolic gaps). The algorithm is based on the principle of using diverse information, such as enzyme and reaction annotations, and experimental data (such as metabolomics), to selectively increase the metabolic knowledge of an organism's existing curated genome-scale metabolic network. ReFill makes use of a repository of reactions, in this case KEGG reaction annotations for MED4 absent from the model, to construct all potential chains of reactions connecting two metabolites in the existing network. It systematically tests the potential of adding each new reaction and suggests adding it only if it can be a part of a chain in which all the metabolites are part of a path in the network (Figure 2). This prevents 


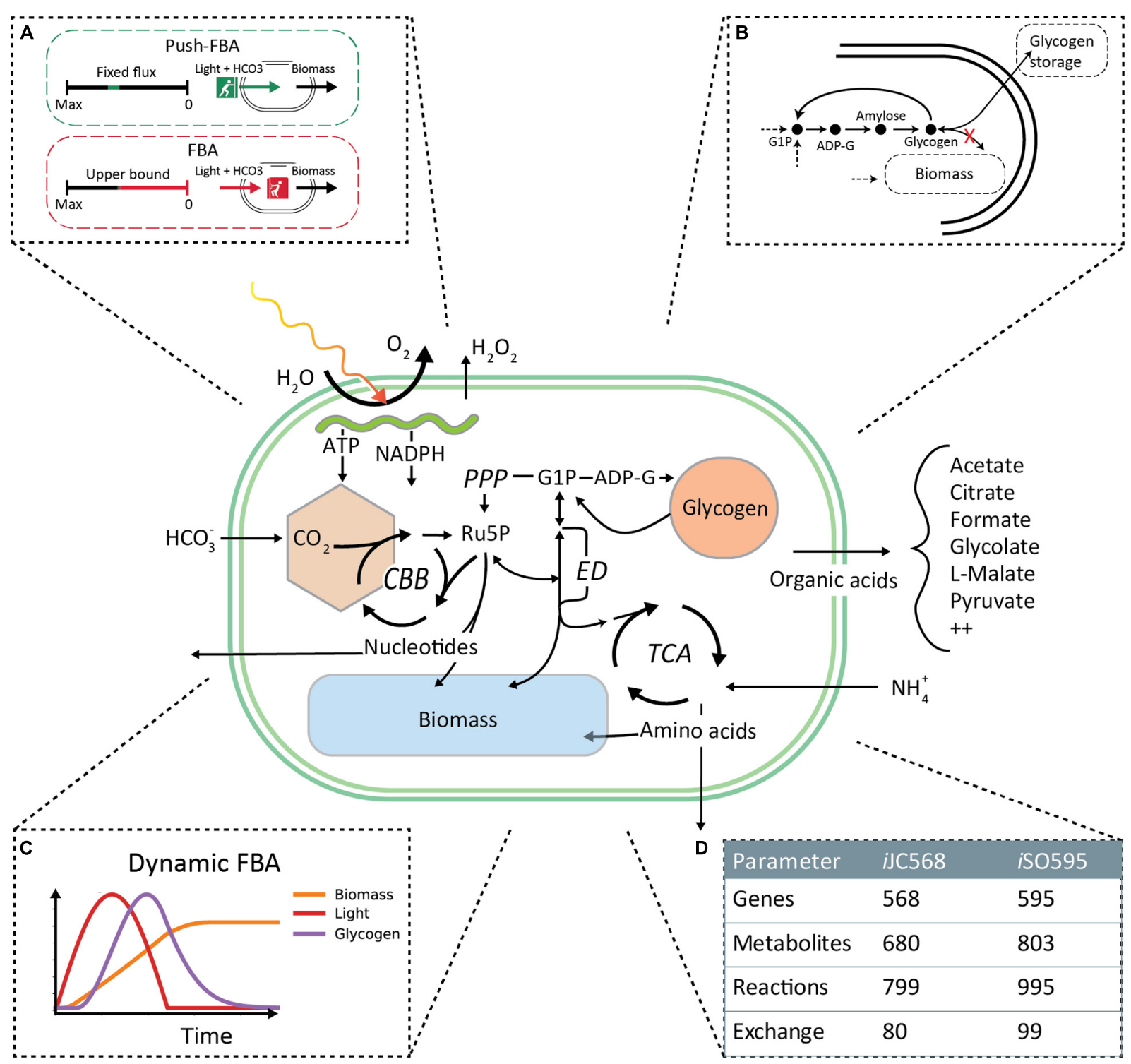

FIGURE 1 | iSO595 is an updated reconstruction of Prochlorococcus marinus MED4, featuring a complete Entner-Doudoroff pathway, rewired glycogen metabolism and increased coverage of the genome. The central panel is a simplified illustration covering the most relevant metabolic features. (A) To simulate the natural environmental constraints experienced by Prochlorococcus we use "Push-FBA": Light and bicarbonate uptake are given a fixed flux independent of growth rate. This contrasts standard FBA where light and bicarbonate would be "pulled" in by the demand needed to support maximal growth rate up to a given bound. (B) We rewired the glycogen metabolism in iSO595 to study the dynamic allocation the dynamic allocation of glycogen. (C) Additionally, we implemented dynamic light conditions and light absorption in COMETS to simulate the growth of $P$. marinus during the diel cycle. (D) iSO595 has increased coverage both in terms of genes, reactions and metabolites compared to its ancestor iJC568.

the creation of new orphan metabolites and potential blocked reactions. The algorithm starts by selecting a reaction from the repository. It then inspects each metabolite in the reaction for presence in the existing network. In case a metabolite is not present, the set of available reactions is scanned for other reactions using this metabolite as a substrate or product. If such a reaction is found, it is added to the chain of potential reactions. The algorithm then iteratively expands the chain until either the repository is exhausted or all the metabolites in the most recent reaction added are present in the network. After all the possible chains of new reactions are expanded, the algorithm examines the connectivity of all the metabolites in each chain (see example in Figure 2B). Following the manual addition of transporters found through TransportDB (Elbourne et al., 2017) and Metabolights (Haug et al., 2013) (Study MTBLS567), using the ReFill algorithm, we updated reactions that belong to several different pathways, including metabolism of cofactors and vitamins, carbohydrate metabolism, amino acid metabolism and nucleotide metabolism. A complete list of added reactions can be found in Supplementary Table 1. ReFill was coded in python 


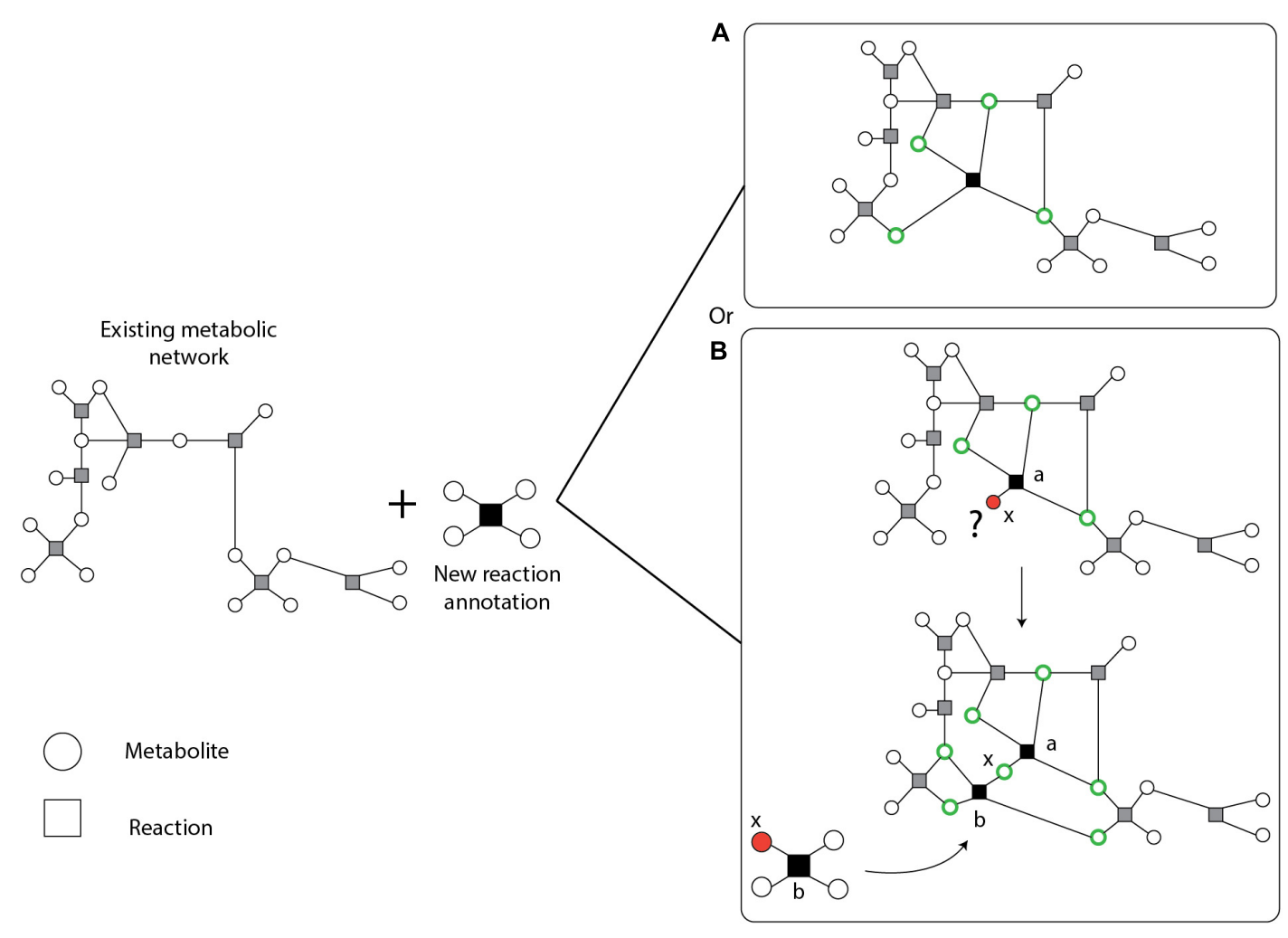

FIGURE 2 | Schematic description of the ReFill algorithm. New reaction annotations are added only if all the metabolites are connected to the existing network. A connected metabolite is denoted in a green circle. An orphan (unconnected metabolite) is denoted in a red circle. (A) A simple case in which all the metabolites comply with the selection rule. (B) In case one or more metabolites are not connected to the existing network, other new reactions may be added to complete the missing connections. For example, consider reaction a to be composed of two substrates and two products, one of which, $x$ (a metabolite that did not exist in the initial network), is not connected to the existing network and is currently an orphan. After the expansion step, the algorithm identified one reaction, $b$, in which $x$ is used as a substrate. All other metabolites in $b$ exist in the network thus creating a path from reaction a through reaction $b$ to the network.

3.7 and generates MATLAB-compatible files formatted to be used with the COBRA Toolbox (Heirendt et al., 2019), including a list of suggested reactions to add and their gene-reaction rules. Other outputs include the added reaction chains and possible metabolic circuits that can be formed by these additions.

\section{Parameter Sampling}

To study the effects of combinations of key nutrients on glycogen production and exudation in the iSO595 model we focused on four parameters representing the uptake fluxes of light, bicarbonate, ammonium, and phosphate. Light and inorganic carbon (bicarbonate) are the substrates for photosynthesis, whereas nitrogen and phosphorus limit the growth of Prochlorococcus in large regions of the world ocean (Davey et al., 2008; Moore et al., 2013; Saito et al., 2014), and nutrient limitation is likely to influence the exudation of fixed carbon (Dubinsky and Berman-Frank, 2001). We sampled 10,000 different environmental conditions by drawing random values from uniform distributions of these four parameters. The range of each parameter was based on physiologically relevant ranges we extracted from the literature and on the requirement that each range covers important phase transitions, such as nutrient and light limitations (Supplementary Table 3).
Light flux was converted from micromole quanta $\mathrm{m}^{-2} \mathrm{sec}^{-1}$ to mmol gDW ${ }^{-1} \mathrm{~h}^{-1}$ similarly to Nogales et al. (2012) using $8 \%$ photosynthesis efficiency rate (Zinser et al., 2009). All uptake flux parameters are described in FBA-compatible units (mmol $\mathrm{gDW}^{-1} \mathrm{~h}^{-1}$ ), while corresponding values in biogeochemistry relevant units are illustrated in Supplementary Table 3. As MED4 is a photoautotroph, it is exposed to a constant stream of light during daylight hours. The bacterium is then forced, or 'pushed,' to fix carbon even when there is not enough of other elements, such as nitrogen or phosphate, to combine the fixed carbon into biomass. To capture this phenomenon in silico we developed a 'push'-FBA framework where we fixed both upper and lower uptake rates of light and bicarbonate (Figure 1A). For the other sampled nutrients, ammonium and phosphate, we defined standard FBA bounds where the maximal uptake rate was set to the sampled value and the lower bound was set to zero. Note that we only considered uptake of sulfur in the form of sulfate (not hydrogen sulfide), and no upper limit was set for the uptake of sulfate because of its abundance in seawater. The maximum rate of RuBisCO (R00024) was fixed to $4.7 \mathrm{mmol}$ $\mathrm{gDW}^{-1} \mathrm{~h}^{-1}$, as previously reported (Casey et al., 2016). Before sampling we blocked a set of artificial exchange reactions that were added in the previous version of the model, most likely 
to allow export of dead-end metabolites that would otherwise limit flux feasibility (Supplementary Table 4). Subsequently, we removed all unconditionally blocked reactions in the model to speed up computations. For each random sample, we first tested the model for feasibility using FBA (Varma and Palsson, 1994). If the solver returned a solution that was feasible and optimal, we further calculated optimal fluxes with parsimonious FBA (Lewis et al., 2010), and determined the range of possible fluxes at optimum with Flux Variability Analysis (FVA) (Gudmundsson and Thiele, 2010). Exchange fluxes from FBA, parsimonious FBA and FVA were recorded and used in subsequent analyses. All environmental sampling and calculations were performed using CobraPy (Ebrahim et al., 2013) and GUROBI 8.1.1 (Gurobi Optimization, Inc., Houston, TX, United States).

\section{Statistical Analysis of the Sampled Spaces}

We sampled 10,000 different environmental conditions based on the flux ranges described above, and analyzed the results of FBA optimization, with the goal of characterizing the distribution of, and correlation between, specific exchange (import/export) fluxes. To that end, we calculated Pearson correlations between exchange reaction fluxes in the sampling data using the python (version 3.7) Pandas package version 1.0.3 (McKinney, 2010). While negative values are normally used to define uptake in FBA, we converted them to positive values for the uptake of light, bicarbonate, phosphate, ammonium, and sulfate when calculating correlations to ease interpretation of the results. We also performed hierarchical clustering using the Nearest Point Algorithm in SciPy (Virtanen et al., 2020) to sort the order of the compounds in the correlation matrix.

We performed dimensionality reduction on normalized exchange reaction fluxes using the T-distributed Stochastic Neighbor Embedding (t-SNE) method (van der Maaten and Hinton, 2008) in Scikit-learn (Pedregosa et al., 2011) with perplexity of 50 and 3,000 iterations. The reaction fluxes were normalized to $[-1,1]$ by dividing by the maximum absolute flux value of each reaction to ensure a consistent influence on the t-SNE results from the different exchange reactions. We considered other normalization schemes, in particular standardization, but found that it was preferable not to center the data to easily discriminate uptake and exudation without further modifications in subsequent data visualization. Finally, the t-SNE transformed data was clustered using HDBSCAN (McInnes et al., 2017) with a minimum cluster size of 200. Transport of inorganic ions, water, and protons were not considered when calculating correlations, dimensionality reduction or clustering. We also discarded transport reactions with no absolute flux value above $10^{-3} \mathrm{mmol} \mathrm{gDW}^{-1} \mathrm{~h}^{-1}$ in any of the environmental samples.

\section{Dynamic Modeling of Light Absorption During the Diel Cycle in COMETS}

Cyanobacteria follow a diel cycle. To capture this dynamic behavior, we extended the Computation Of Microbial Ecosystems in Time and Space (COMETS) platform (Harcombe et al., 2014; Dukovski et al., 2020), and developed a module for diurnalcycle simulations allowing oscillations of light intensity and light absorption. Attenuation of light through each grid cell was modeled using the Beer-Lambert law, as described previously (Yang, 2011; Gomez et al., 2014):

$$
I(t, z)=I_{0}(t) e^{-\left(a_{w}+a_{d w} X(t)\right) z}
$$

Here, $I(t, z)$ is the light irradiance given in mmol photons $\mathrm{m}^{-2} \mathrm{~s}^{-1}, t$ is the time, $z$ is the depth (from the top of the grid cell), $a_{\mathrm{dw}}$ is the cell- and wavelength-specific absorption coefficient given in $\mathrm{m}^{2} \mathrm{gDW}^{-1}, a_{\mathrm{w}}$ the absorption coefficient of pure water given in $\mathrm{m}^{-1}, X(t)$ the biomass concentration in $\mathrm{gDW} \mathrm{m} \mathrm{m}^{-3}$, and $I_{0}(t)$ the time-dependent incident light irradiance at the top of the grid cell. In the current version, we simplified the process by assuming that the light irradiance is either monochromatic or a sum of the total light bandwidth, and the absorption coefficient should match the wavelength(s) of the light source. The total light attenuation $(\Delta I)$ through a grid cell of thickness $\Delta z$ is then

$$
\Delta I(t)=I(t, 0)-I(t, \Delta z)=I_{0}\left(1-e^{-\left(a_{w}+a_{d w} X(t)\right) \Delta z}\right)
$$

The light absorbed by the cells is a fraction of the total light attenuation, i.e.,

$$
I_{\mathrm{abs}}(t)=\frac{\Delta I(t) \cdot a_{\mathrm{dw}} X(t)}{a_{\mathrm{w}}+a_{\mathrm{dw}} X(t)} .
$$

The total number of photons absorbed per dry cell weight $[\Phi(t)]$ in mmol photons $\mathrm{gDW}^{-1} \mathrm{~s}^{-1}$ by the cells within a grid cell of thickness $\Delta z$, volume $V$, and surface area $A$ is then

$$
\Phi(\mathrm{t})=\frac{I_{\mathrm{abs}}(t) \cdot A}{X(t) \cdot V}=\frac{I_{0}(t)}{\Delta z} \frac{a_{\mathrm{dw}}}{a_{\mathrm{w}}+a_{\mathrm{dw}} X(t)}\left(1-e^{-\left(a_{\mathrm{w}}+a_{\mathrm{dw}} X(t)\right) \Delta z}\right) .
$$

For all COMETS simulations presented here we have used monochromatic light at $680 \mathrm{~nm}$ with a calculated biomassspecific absorption coefficient $a_{\mathrm{dw}}$ as previously described (Morel and Bricaud, 1981; Bricaud et al., 2004). Briefly, the biomassspecific absorption is the weighted sum of the absorption coefficients of the light-absorbing pigments divinyl-chlorophyll $\mathrm{A}$ and $\mathrm{B}$, since none of the other pigments in Prochlorococcus absorb light at $680 \mathrm{~nm}$. Additionally, to account for the discrete distribution of chlorophyll into separate cells, the absorption coefficient is scaled by the packaging factor. All coefficients used to calculate light attenuation and absorption are provided in Table 1.

The changing light conditions throughout a diel cycle was modeled as

$$
I_{0}(t)=A \max (\sin (\omega t), 0),
$$

where the angular frequency is $\omega=\frac{2 \pi}{T}$.

Following the development of the diel cycle simulation capability in COMETS we set out to dynamically simulate the growth of MED4. Since the nutrient uptake follows MichaelisMenten kinetics, we estimated the kinetic parameters $V_{\max }$ and $K_{\mathrm{m}}$ using a heuristic approach from experimental data 
TABLE 1 | Coefficients and values used to calculate light absorption in COMETS.

\begin{tabular}{|c|c|c|c|c|}
\hline Symbol & Description & Value & Unit & Reference \\
\hline$\lambda$ & Wavelength & 680 & $\mathrm{~nm}$ & \\
\hline$a_{w}$ & Absorption coefficient of water at $680 \mathrm{~nm}$ & 0.465 & $m^{-1}$ & Pope and Fry, 1997 \\
\hline$a_{d w}$ & Biomass-specific absorption coefficient & 0.285 & $\mathrm{~m}^{2} \mathrm{gDW}^{-1}$ & \\
\hline$a_{d v c h l-A}$ & Absorption coefficient of divinyl-chlorophyll A at $680 \mathrm{~nm}$ & 0.0184 & $m^{2}(m g d v c h l-A)^{-1}$ & Bricaud et al., 2004 \\
\hline$a_{d v c h l-B}$ & Absorption coefficient of divinyl-chlorophyll B at $680 \mathrm{~nm}$ & 0.0018 & $m^{2}(m g d v c h l-B)^{-1}$ & Bricaud et al., 2004 \\
\hline $\mathrm{C}_{d v c h l-A}$ & Amount of divinyl-chlorophyll A & 0.0163 & g dvchl-A gDW ${ }^{-1}$ & Casey et al., 2016 \\
\hline$C_{d v c h l-B}$ & Amount of divinyl-chlorophyll B & 0.0013 & $\mathrm{~g} \mathrm{dvchl}-\mathrm{B}_{\mathrm{gDW}}{ }^{-1}$ & Casey et al., 2016 \\
\hline$d$ & Average diameter of MED4 & 0.6 & $\mu \mathrm{m}$ & \\
\hline$n^{\prime}$ & Imaginary part of the refractive index at $675 \mathrm{~nm}$ & 0.01377 & & Stramski et al., 2001 \\
\hline$Q^{*}$ & Packaging effect at $680 \mathrm{~nm}$ & 0.945 & & \\
\hline
\end{tabular}

(Grossowicz et al., 2017), first by finding the range of possible parameter combinations corresponding to the gross growth rate of $0.5 \mathrm{~d}^{-1}$ (Supplementary Figure 1A), and secondly by comparing predicted growth and ammonium depletion with the experimental time-series cultivation data (Supplementary Figures 1B,C). The estimated parameters were used in the remaining dynamic FBA simulations in COMETS. Finally, to simulate the dynamic storage and consumption of glycogen we applied a multiple objective approach consisting of the following four steps: (1) Maximization of the flux through the non-growth associated maintenance reaction. Note that, this reaction has an upper bound of $1 \mathrm{mmol} \mathrm{gDW}^{-1} \mathrm{~h}^{-1}$ (Casey et al., 2016). In contrast to standard practice, where one uses a lower bound for the non-growth associated maintenance reaction, this method provides a more realistic scenario where the organism continues to consume resources trying to keep up cellular maintenance even at zero growth; (2) Maximization of growth; (3) Maximization of glycogen production (storage); and (4) Parsimonious objective which minimizes the sum of absolute fluxes. To simulate nitrogen-abundant and nitrogenpoor growth conditions, we used the PRO99 medium with standard $(800 \mu \mathrm{Mol})$ and reduced $(100 \mu \mathrm{Mol})$ ammonium concentration, as previously described (Grossowicz et al., 2017). Light availability was modeled as described in Equation 5, with an amplitude of $40 \mu \mathrm{mol} Q \mathrm{~m}^{-2} \mathrm{~s}^{-1}$ and a period of $24 \mathrm{~h}$. We also incorporated a death rate of $0.1 \mathrm{~d}^{-1}$, similar to previous modeling efforts on Prochlorococcus (Grossowicz et al., 2017). All parameter values used in the COMETS simulations are given in Supplementary Table 5. All dynamic growth simulations were performed using COMETS v.2.7.4 with the Gurobi 8.1.1 solver, invoked using the associated MATLAB toolbox ${ }^{1}$.

\section{Simulating Growth of Knockout Mutants}

Simulations of the knockout mutants where performed by constraining the flux to zero for the reactions catalyzed by the enzymes encoded by $g l g C$ (PMM0769) and gnd (PMM0770), respectively. For $g \lg C$, the reaction is glucose-1-phosphate adenylyltransferase (R00948) and for gnd the two reactions are $\mathrm{NADP}^{+}$and $\mathrm{NAD}^{+}$associated 6-phosphogluconate

${ }^{1}$ https://github.com/segrelab/comets-toolbox dehydrogenases (R01528 and R10221). We then used dynamic FBA in COMETS with PRO99 medium (Moore et al., 2007) with limited ammonium and diel light conditions to simulate growth over 7 days. The growth curves where qualitatively compared with experimental data from Shinde et al. (2020).

\section{RESULTS AND DISCUSSION}

\section{Model Curation and Update}

Prochlorococcus fixes carbon through photosynthesis during daytime. Fixed carbon that is neither used for cell growth nor stored in the form of glycogen is exuded. Here, we set out to study dynamic changes in the carbon allocation and storage mechanisms in MED4 using a genome-scale metabolic modeling approach. To that end, we first re-curated and updated the available iJC586 model (Casey et al., 2016), as described in detail in the "Materials and Methods" section. The update involved the development of a new semi-automatic algorithm (ReFill), which can be broadly applied to other reconstructions (see section Materials and Methods). Concurrently, we introduced a revised mechanism for carbon storage, effectively treating glycogen as an independent component of biomass. This dynamic implementation of glycogen storage, introduced here in $\mathrm{dFBA}$, makes it possible for glycogen to be accumulated and depleted at variable rates (Figure 1), aligning with the overflow metabolism hypothesis (Szul et al., 2019; de Groot et al., 2020). Other key modifications induced by the ReFill algorithm and subsequent manual curation (see section Materials and Methods) include the completion of the Entner-Doudoroff (ED) pathway, recently discovered in cyanobacteria (Chen et al., 2016) and proposed as the primary Prochlorococcus glucose metabolism pathway under mixotrophic conditions (Biller et al., 2018; Muñoz-Marín et al., 2020). Additional revisions focused on the exudation of fixed carbon products from the cell and included various transports such as pyruvate, fumarate, citrate, ethanol, various nucleotides and hydrogen peroxide as well as metabolites found in both the endo- and exo- metabolome of Prochlorococcus (Metabolights study MTBLS567). The end product of our revision, reconstruction $i$ SO595, has 595 genes, 802 metabolites and 994 reactions, i.e., 27 genes, 123 metabolites and 196 reactions more than the previous version, iJC568 (Figure 1D). 


\section{Carbon Fixation and Storage Are Affected by Nutrient Uptake Rate}

Prochlorococcus thrive in oligotrophic environments (Johnson et al., 2006), where, in surface waters, its growth and carbon fixation rates are usually limited by the abundance of nitrogen, phosphate or iron (Krumhardt et al., 2013; Saito et al., 2014; Szul et al., 2019). Deeper in the water column Prochlorococcus growth becomes limited by light (Vaulot et al., 1995). We set out to explore the combined effect of different levels of light and nutrients on carbon fixation, storage and exudation. Similarly to Phenotypic Phase Plane analysis (Edwards et al., 2002), we sought a global perspective of metabolism in this multi-parameter spaces while explicitly taking into account the fact that the inflow of light and bicarbonate may not be easily controllable by the cell, and that Prochlorococcus may need to deal with excess amounts of fixed carbon. Thus, in contrast to normal FBA where the uptake of metabolites is constrained by an upper bound, we introduced a 'push- FBA' approach (Figure 1A), in which the influx of bicarbonate and light have a fixed imposed value (see section "Materials and Methods" and Supplementary Table 3 for specific values used). This approach attempts to mimic implications of photosynthesis, in which light is the driving force. Once photons are absorbed by the chlorophyll in the photosynthetic reaction centers, most of the energy must be used to produce ATP and reducing power, otherwise it is dissipated in ways that may cause cell damage (Long et al., 1994). We note that this modeling approach over-simplifies the complex process of photosynthesis; for example, we do not account for the dynamics of photoprotective pigments, which allow some of the incident photons to be dissipated as heat. Indeed, the ratio of the photoprotective pigment zeaxanthin to divinyl chlorophyll $a$ increases under nitrogen starvation, suggesting that, under these conditions, some of the photon flux may be diverted from the reaction centers (Steglich et al., 2001; Roth-rosenberg et al., 2019). Nevertheless, Prochlorococcus undergo photoinhibition at high light intensities (Moore et al., 1995; Mella-Flores et al., 2012), despite the presence of photoprotective pigments and other protection mechanisms such as cyclic electron flow [which is represented in the model (Casey et al., 2016)]. Thus, these mechanisms do not allow the cell to fully control the flux of photons through the photosystem and the resulting fluxes in ATP and reducing power, in a manner that is reflected in the push-FBA approach. This subtle difference in applied constraints has major effects on model predictions. While flux rearrangement is usually viewed as a consequence of environmental nutrient limitations, the results of this analysis show that a substantial rewiring of fluxes is caused by this imposed excess of fixed carbon as well.

To understand how different combinations of environmental parameters (availability of nitrogen, phosphate, light and bicarbonate) affect the way Prochlorococcus can manage its carbon budget, we implemented FBA under 10,000 randomly sampled growth environments. Overall, this sampling analysis demonstrated that the exudation of organic acids, aminoacids, and nucleobases/nucleosides, as well as the extent of glycogen storage, are strongly modulated by environmental factors (Figure 3). To observe the full range of possible optimal solutions per sample, we implemented and compared different flux balance analysis methods, including flux variability analysis (FVA) and parsimonious FBA (pFBA). These two methods provide complementary insight: FVA estimates the range of possible values for the flux of each reaction at the optimum, providing insight into the structure of the phenotypic space at maximal growth rate. In contrast, $\mathrm{pFBA}$, by minimizing the sum of fluxes at optimality, generates flux predictions less likely to involve unrealistic loops, and thus potentially provides predictions closer to experimental values (Lewis et al., 2010). Together, these two FBA methods help analyze the solutions of our high-dimensionality dataset.

Our predictions simulate the metabolic effects and variability in glycogen production modulated by environmental constraints (Figure 3). Glycogen production was observed only above light levels of $50 \mathrm{mmol} \mathrm{gDW}^{-1} \mathrm{~h}^{-1}$ (corresponding to 7.5 micromole quanta $\mathrm{m}^{-2} \mathrm{sec}^{-1}$ ), and decreased as ammonium and phosphate concentrations increase. These observations do not contradict previous evidence showing increased glycogen accumulation in faster growing cyanobacteria (Zavřel et al., 2019), rather they align with previous studies finding that glycogen storage is enhanced in nutrient-limiting conditions (Monshupanee and Incharoensakdi, 2014; Szul et al., 2019). Interestingly, FVA consistently predicted the glycogen production range minimal value to be zero across all samples. This implies that glycogen storage is possible, but not necessary to achieve optimal growth in the feasible solution space. This was also the case in the more stringent $\mathrm{pFBA}$ analysis, indicating that while metabolism may be a strong modulator of glycogen metabolism, more types of regulation, not accounted for in FBA, are involved. One example of such regulation may be allosteric regulation of ADP-glucose pyrophosphorylase by 3-phosphoglycerate (Iglesias et al., 1991), possibly in combination with redox regulation (Díaz-Troya et al., 2014). Specific regulation aimed at tuning up glycogen storage may also occur at the transcriptional level, e.g., by multiple transcription factors previously suggested to be involved in the regulation of glycogen metabolism in fluctuating environments (Luan et al., 2019).

The range of possible rates of glycogen production (through FVA) displays a bell-shaped bicarbonate-dependent distribution, indicating low storage of glycogen (zero flux) under both low and high uptake rates of bicarbonate. When bicarbonate uptake rates are low, all available carbon is diverted into growth. The reduced glycogen storage at high bicarbonate uptake, when $\mathrm{RuBisCO}$ is saturated, seems to be caused by the increased ATP demand associated with the conversion of bicarbonate to exudation-products, since the onset and rate of change of this trade-off is modulated by the ATP availability, as demonstrated by phenotypic phase planes analysis (Supplementary Figure 2). This agrees with recent work suggesting that Prochlorococcus use available ATP to drive pathways to saturation by shifting reaction directions toward favoring dephosphorylation of ATP to ADP, disrupting the cellular ATP/ADP ratio and increasing the metabolic rate of the cell by pushing forward ATP consuming reactions, until it is restored. Together with organic carbon exudation this strategy allows for growth in lower nutrient concentrations (Braakman, 2019). 


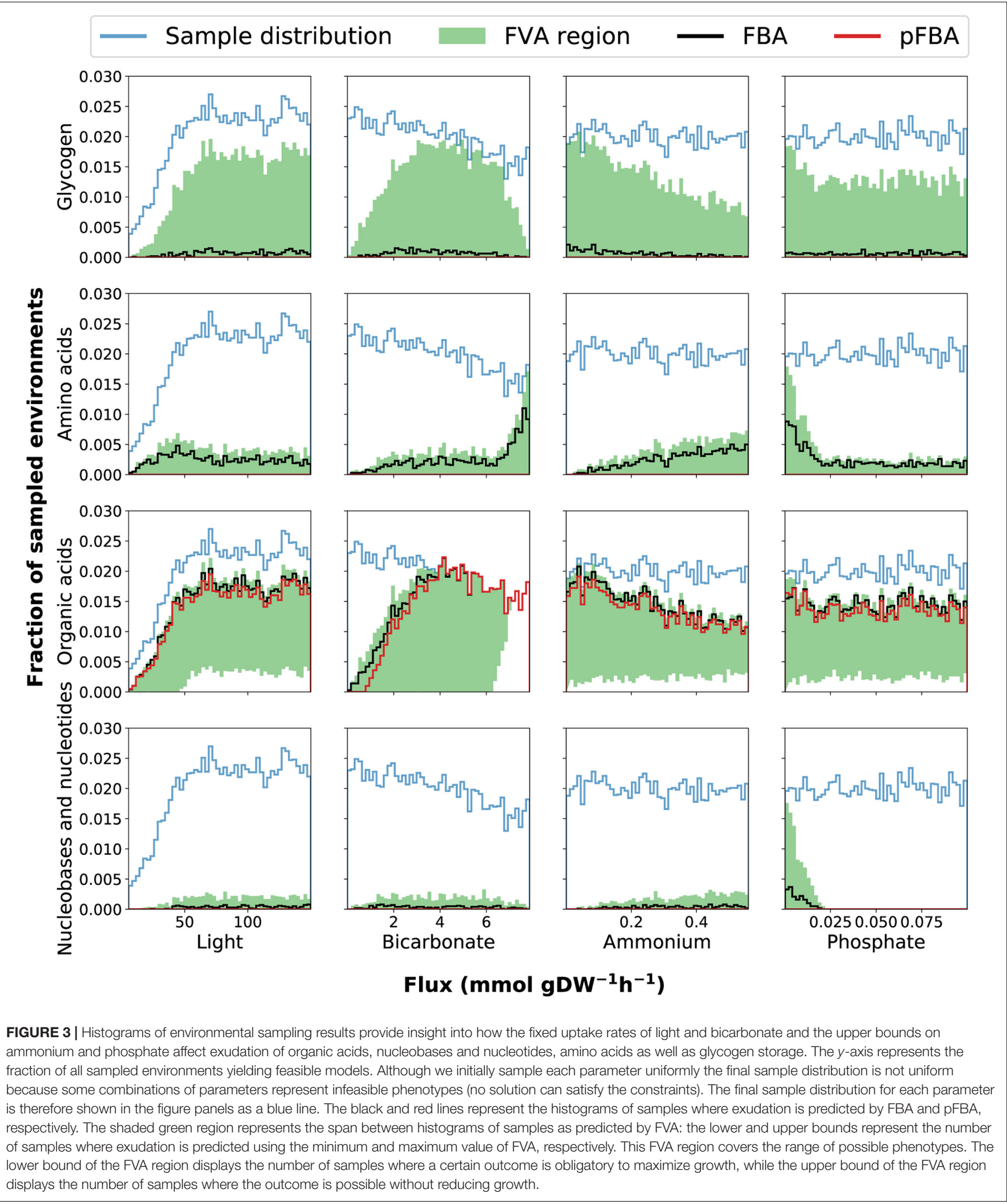

We next sought to explore the effect of combinations of key nutrients on storage and exudation patterns in our sampling spaces. To that end, we visualized the data using t-SNE clustering
(Figure 4A). To explore the strongest trends, we chose to employ a high stringency approach and use only our set of pFBA results in this context. Due to the nature of pFBA, 

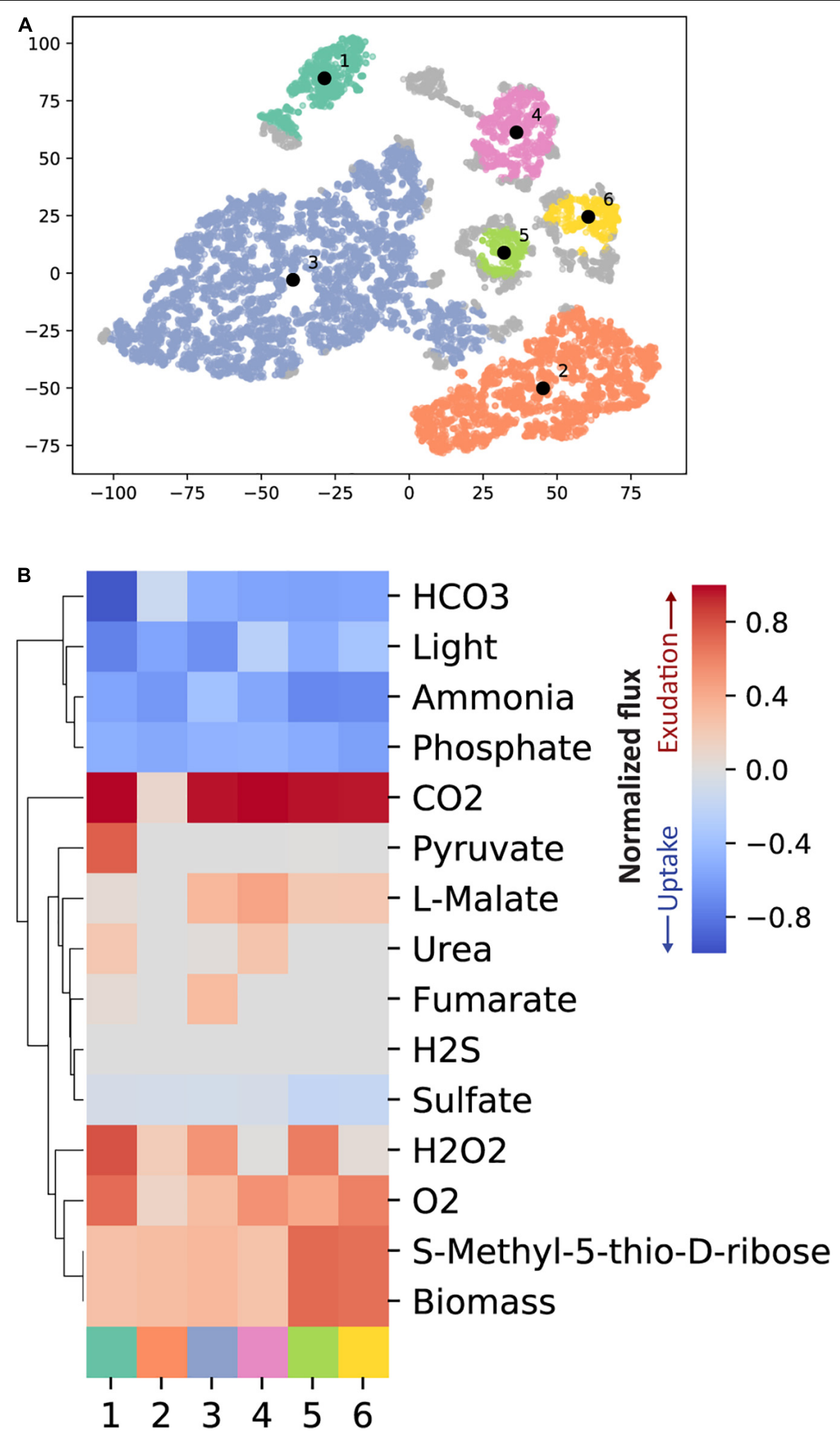

FIGURE 4 | T-SNE clustering identifies typical phenotypes from the pFBA results from the random samples. (A) The random samples are reduced into two dimensions with t-SNE. We have subsequently used HDBSCAN to cluster the data. HDBSCAN identified six disjoint clusters which represent different phenotypes. (B) For each of the six clusters the mean uptake or exudation across all samples within the respective cluster is shown. Only exchange reactions with an absolute flux above $1 \mathrm{e}-3 \mathrm{mmol} g D W^{-1} \mathrm{~h}^{-1}$ in any of the random samples are included. 
any exudation observed in this analysis could not be easily removed without imposing a cost on growth. We observed 6 typical phenotypes (clusters) rising out of the sampling spaces (Figure 4). These 6 phenotypes are characterized by subtle differences in combinations of environmental parameters, yielding significantly different exudation patterns. Generally, we observed the highest biomass value in phenotype 5, and the lowest in phenotype 4. All key nutrient uptake rates were highly variable (ranging from 33 to $44 \%$ variability). Phenotype 1 is characterized by high light, bicarbonate, a maximum RuBisCO flux (indicating maximal photosynthesis rate) but low nitrogen uptake. Additionally, we observed high exudation of pyruvate coming from the pentose phosphate and Entner-Doudoroff pathways. Both are alternative routes coming out of carbon fixation (Waldbauer et al., 2012; Chen et al., 2016). Together with a low biomass value, this phenotype might indicate a scenario of exudation due to overflow metabolism.

The two largest clusters (numbers 2 and 3, Supplementary Figure 3), tie together high and low light, carbon and nitrogen uptake rates, and different exudation patterns. Interestingly, phenotype 3 (high light) showed exudation of fumarate and malate while phenotype 2 (low light) did not. Recent work suggested that, in high light conditions, fumarate is generated through oxaloacetate and malate creating a broken acyclic form of the TCA cycle, while in the dark, fluxes are diverted into forming the cyclic form of it. This low light form of the TCA cycle is then active and works toward energy generation (Xiong et al., 2017). Similarly, we observed two forms of the TCA cycle in the high and low light phenotypes (2 and 3, respectively) with a difference in the direction of one reaction (KEGG R00342, Supplementary Figure 3). Phenotype 2, describing lowlight conditions, showed the L-Malate/oxaloacetate balance to shift in favor of oxaloacetate, completing the route toward 2Oxoglutarate, a key metabolite known to act as a starvation signal and modulator of the $\mathrm{C} / \mathrm{N}$ balance in cyanobacteria (DomínguezMartín et al., 2018; Zhang et al., 2018), and subsequently into energy generation. On the other hand, Phenotype 3, describing high light conditions, showed the L-Malate/oxaloacetate balance to shift in favor of L-Malate and away from the formation of 2-oxoglutarate. In both phenotypes fumarate is converted to LMalate. While in Phenotype 2 it is fed into a semi-cyclic form of the TCA cycle, fumarate is partly exuded and partly converted to L-malate in phenotype 3 , in agreement with overflow metabolism.

We observed a similar TCA cycle flux distribution in phenotype 4 as in phenotype 3, leading to high exudation of L-Malate. Interestingly, Phenotype 1 and 4 are comparable in all key nutrients except light (High in phenotype 1 and low in phenotype 4). As a result of an in-depth flux distribution analysis, we observed a reaction direction change in UDP-glucose:NAD+ 6-oxidoreductase [R00286, EC 1.1.1.22, PMM1261] between the two phenotypes. In phenotype 4 this reaction shifted toward the creation of UDP-glucose, a precursor for the production of glycogen (due to the high stringency of this analysis we did not observe the direct formation of glycogen). In phenotype 1, this reaction favored the formation of UDP-glucuronate which in turn was diverted into the formation of amino sugars. These phenotypes may correlate to the 12:00 (phenotype 1) and 16:00 (phenotype 4) scenarios described in Szul et al. (2019). Finally, Phenotypes 5 and 6 may represent a high-light nutrient-rich environment resulting in a high biomass value.

\section{Nutrient Uptake Rates Modulate Exudation of Organic Compounds}

The use of genome-scale metabolic models captures a comprehensive picture of the metabolic processes taking place in the cell, including those that lead to metabolite exudation. From the random sampling of environmental conditions, we identified conditions in which organic acids must be exuded. This was noticeable by a non-zero lower bound of the FVA region (Figure 3). Interestingly, organic acids were more likely to be exuded when the growth became limited by phosphate or nitrogen. Since Prochlorococcus is known to thrive in oligotrophic ocean gyres where nitrogen or phosphate is limited (Partensky et al., 1999; Flombaum et al., 2013), this represents a likely natural phenotype, and as such, supports previous findings (Bertilsson et al., 2005; Szul et al., 2019). Costly metabolites, essential for cell survival and growth, such as amino acids, nucleobases and nucleotides, tend to be exuded in nitrogen and carbon rich conditions and might be a result of overflow metabolism (Cano et al., 2018; Pacheco et al., 2018). To explore this phenomenon in further detail, we looked into exudation patterns of specific metabolites as a function of key nutrient limitations (Figure 5). Of the environmental factors, the uptake of nitrogen (ammonium) is a decisive factor differentiating between exudation of organic acids or amino acids. While it is positively correlated with the exudation of nitrogen-rich compounds such as amino acids, it is negatively correlated with exudation of organic acids and glycogen. Additionally, glycogen formation is positively correlated with the exudation of malate, citrate, fumarate, and succinate, which are most of the TCA cycle constituents. This is in line with previous findings suggesting the re-direction of carbon metabolism toward the formation of macromolecules (including glycogen) in nitrogen limiting conditions (Forchhammer and Selim, 2019; Szul et al., 2019). Thus, our reconstruction captured known possible aspects of the carbon/nitrogen balance in Prochlorococcus.

Finally, we observed a general pattern of strong positive correlations between amino acids, nucleobases, nucleosides, as well as a range of other compounds. In an interesting deviation from this general pattern, L-aspartate showed a decreased correlation with other exudates. L-aspartate, together with its role in protein nucleic acid biosynthesis, can serve as a precursor for nitrogen storage metabolites such as polyamines (Szul et al., 2019). Indeed, we observed a slightly stronger correlation between L-aspartate and the uptake of nitrogen compared to other amino acids. Finally, In contrast to other amino acids, L-aspartate is negatively correlated with light uptake and hydrogen peroxide exudation. Hydrogen peroxide is produced from L-aspartate and oxygen by L-aspartate oxidase [R00481, EC 1.4.3.16, PMM0100]. L-amino acid oxidases have been previously described in cyanobacteria and have been related to the use of amino acids as carbon sources (Campillo-Brocal et al., 2015). The production of hydrogen peroxide is also strongly correlated with 


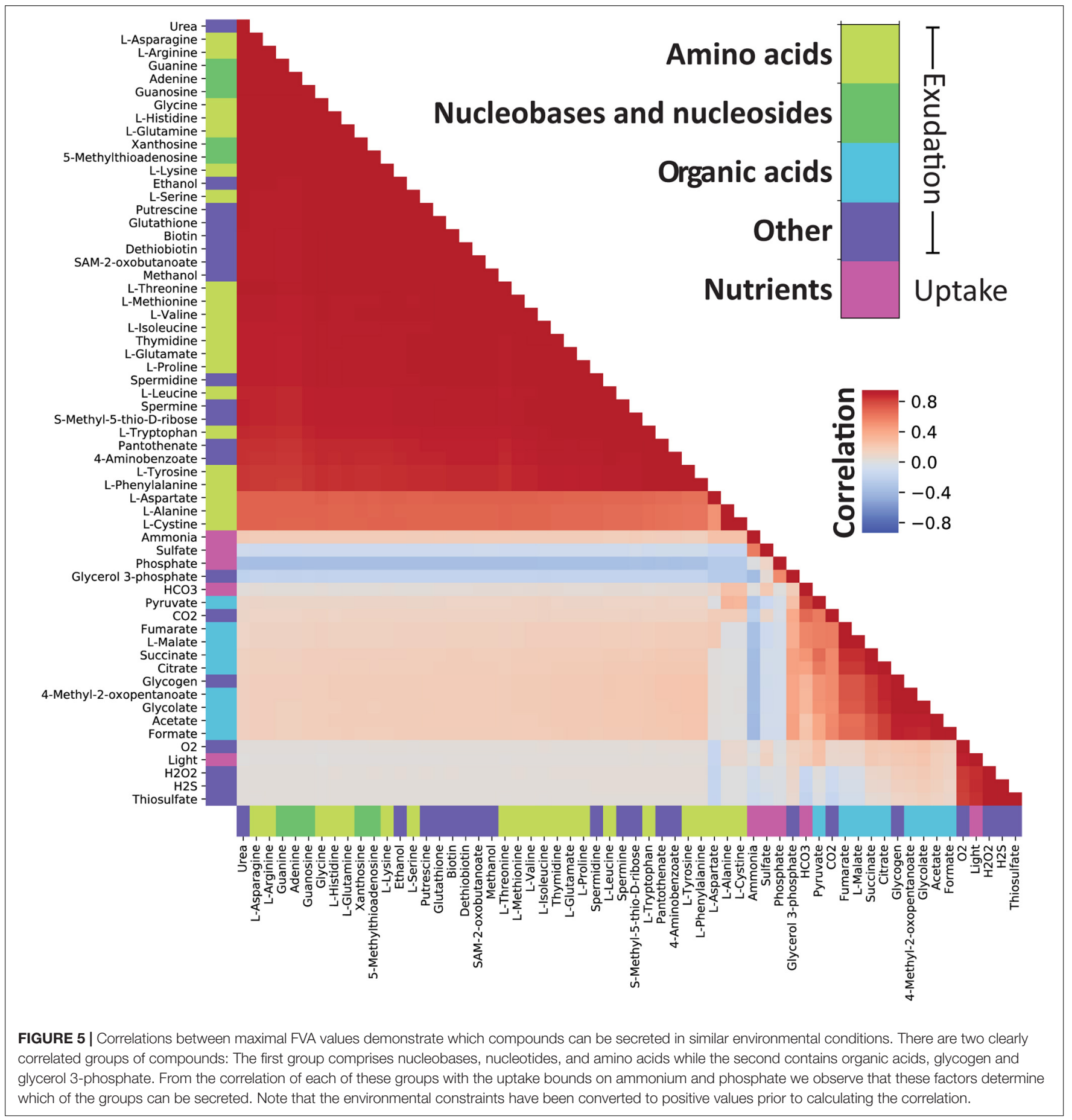

light, a result consistent with the expectation that reactive oxygen species are created during photosynthesis.

\section{Dynamic Allocation of Carbon Storage}

Nutrient and light limitations are well-known modulators of carbon storage in Prochlorococcus (Zinser et al., 2009; Szul et al., 2019). Recent work has suggested the storage of carbon to be one of the major metabolic tasks during the day-night cycle (Cano et al., 2018; Szul et al., 2019; Shinde et al., 2020).
To explore time-modulated trade-offs and trends related to carbon storage, we performed in silico dynamic FBA diel-cycle simulations using the Computational of Microbial Ecosystems in Time and Space (COMETS) platform (Harcombe et al., 2014; Dukovski et al., 2020). COMETS is a population-based dynamic FBA implementation that can simulate growth of millions of cells, but it is important to note that the framework assumes continuous growth on a mesoscopic scale and does therefore not explicitly account for individual cells nor regulated cell 
cycle events such as cell division. COMETS relies on uptake flux kinetic information such as $K_{\mathrm{m}}$ and $V_{\max }$ to simulate the spatial growth and exudation patterns of microbes in a simulated discretized time course. To improve the accuracy and biological relevance of our simulations we used kinetic constants either obtained from experimental measurements reported in the literature (Krumhardt et al., 2013; Hopkinson et al., 2014) (Supplementary Table 3) or from fitting model simulations to measured growth and depletion of ammonium rates (Grossowicz et al., 2017). We found $K_{\mathrm{m}}$ and $V_{\max }$ values of $0.39 \mathrm{mM}$ and $0.9 \mathrm{mmol} \mathrm{gDW}^{-1} \mathrm{~h}^{-1}$ for the uptake of ammonium to best fit the experimental data (Grossowicz et al., 2017) (Supplementary Figure 1). Surprisingly, the estimated $K_{\mathrm{m}}$ value is 3 orders of magnitude larger than previous estimates (Marañón et al., 2013). This deviation might occur due to several reasons. First, our estimates are based on the assumption that growth is indeed limited by the availability of ammonium and that Prochlorococcus operates at a metabolic state close to optimal growth. Other limiting factors or non-optimal growth may lead to incorrect estimates. Nevertheless, it is challenging to fit $K_{\mathrm{m}}$ values accurately from batch cultivation data, as this parameter only becomes dominant in the short time-period immediately prior to nutrient depletion. Furthermore, the accuracy of the fitted $K_{\mathrm{m}}$ value can suffer from the rather high uncertainty in the measured ammonium concentrations, although not more than 2 orders of magnitude (Supplementary Figure 1). Finally, we raise the possibility that Prochlorococcus may possess several ammonium transporters with different affinity as previously observed in marine eukaryotic phytoplankton (McDonald et al., 2010) and cyanobacteria (Kashyap and Singh, 1985). To account for this uncertainty we assessed the sensitivity of our dFBA simulations to variation in the value of $K_{\mathrm{m}}$, in combination with variation in the maximum uptake rate of ammonium $\left(V_{\max }\right)$, ammonium concentration and light intensity (Supplementary Figure 4). The parameters that dictate light absorption (Table 1) affect the number of available photons, so that by including a large span of light intensities in our sensitive analysis, we also cover their associated uncertainty. We find that ammonium concentration, kinetic coefficients for ammonium uptake and the availability of photons combined have a considerable impact on whether carbon is stored during daytime in our dynamic FBA simulations, underpinning the importance of accurate and context specific values for these parameters. This echoes the well-known modulation of carbon storage by nutrient and light limitations (Zinser et al., 2009; Szul et al., 2019). We note that, despite the potentially large impact of Prochlorococcus on marine nitrogen budgets, to the best of our knowledge there are currently no direct experimental measurements of the kinetics $\left(K_{\mathrm{m}}, V_{\max }\right)$ of nitrogen uptake by Prochlorococcus.

Since the tight coupling between carbon and nitrogen metabolism in cyanobacteria is known to influence carbon allocation and storage (Zhang et al., 2018; Szul et al., 2019), it was chosen as a case study. As such, we focused in more detail on the dynamic changes in metabolism in nitrogen-abundant and nitrogen-poor media, as previously defined (Grossowicz et al., 2017). Specifically, we set out to explore glycogen production and consumption with COMETS in these conditions (Figure 6).
We did not observe glycogen storage in nitrogen-abundant simulations, and therefore no growth nor cellular maintenance during nighttime. One explanation for this may arise from the limitations of the platform. First, the simulations performed in this work were performed in a modeling framework based on linear programming with ordered multi-objective optimization: (1) cellular maintenance; (2) growth; (3) glycogen storage. Thus, glycogen was only stored when there were excess energy and carbon available, which occurred when growth was nitrogen limited. Although some observer bias was introduced by assuming that Prochlorococcus is striving toward these cellular objectives, in this order, we found a reasonable conceptual alignment with previous work showing that bacterial metabolism balances a trade-off between maximal growth and the ability to adapt to changing conditions (Schuetz et al., 2012). However, we do note that one might obtain more nuanced results by taking into account suboptimal solutions (Segrè et al., 2002; Fischer and Sauer, 2005; Wintermute et al., 2013), and that real phenotypes may be in the continuum between the two extremes found here. Another limitation that might affect glycogen storage is the lack of regulatory mechanisms not usually accounted for in this version of dynamic FBA (Mahadevan et al., 2002). The addition of regulatory layers or more specifically tailored objective functions, such as global optimization over the entire diel cycle (Reimers et al., 2017), could lead to smaller but non-zero generation of glycogen also during nitrogen-rich conditions.

In agreement with previous work (Szul et al., 2019), under nitrogen-limiting conditions, glycogen accumulates throughout the day and is subsequently used to support respiration and growth during the night (Figure 6A). However, the predicted glycogen storage is simulated as not sufficient to support neither growth nor cellular maintenance throughout the night. This may contribute to the increased death rate during night time (Zinser et al., 2009; Ribalet et al., 2015). However, the rate of glycogen depletion is strongly affected by the associated kinetic parameters (Supplementary Figure 5), emphasizing the value of accurate kinetic coefficients for GlgP, the main contributing factor to glycogen catabolism in bacteria (Dauvillée et al., 2005; Alonso-Casajús et al., 2006; Fu and Xu, 2006), in future work. Furthermore, the rate of glycogen depletion might be modulated by transcriptional regulation. Previous work suggested that glycogen storages are not sustained beyond dawn, because the genes responsible for glycogen degradation are depleted during the first $5 \mathrm{~h}$ of darkness (Biller et al., 2018). Interestingly, the model predicts consumption of glycogen during dusk to increase growth when photosynthesis is declining (Figure 6), closely resembling observations in Synechococcus, in particular for the $\Delta k a i C$ mutant with a dysfunctional circadian clock (Diamond et al., 2015). The closer resemblance of the dysfunctional circadian clock phenotype might be a result from the limitations of the applied modeling framework that does not include regulatory mechanisms.

The switch from photosynthesis at daytime to glycogen consumption at nighttime is reflected in the metabolic shifts observed in key pathways (Figure 6B). Interestingly, we observed higher fluxes through the Calvin cycle in nitrogen-poor conditions. This difference may be caused by the increased 

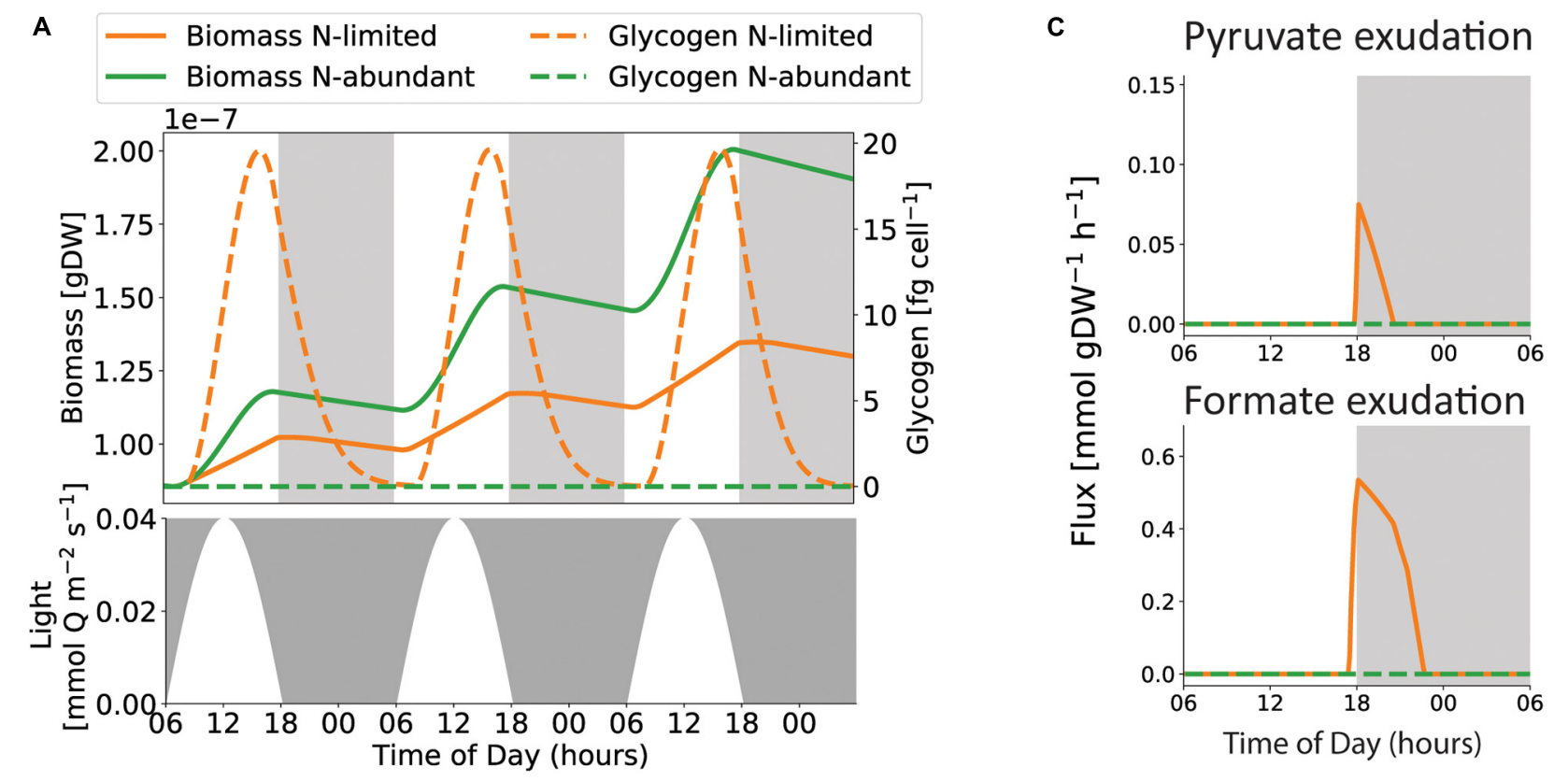

B Nitrogen limited $=-$ - Nitrogen abundant
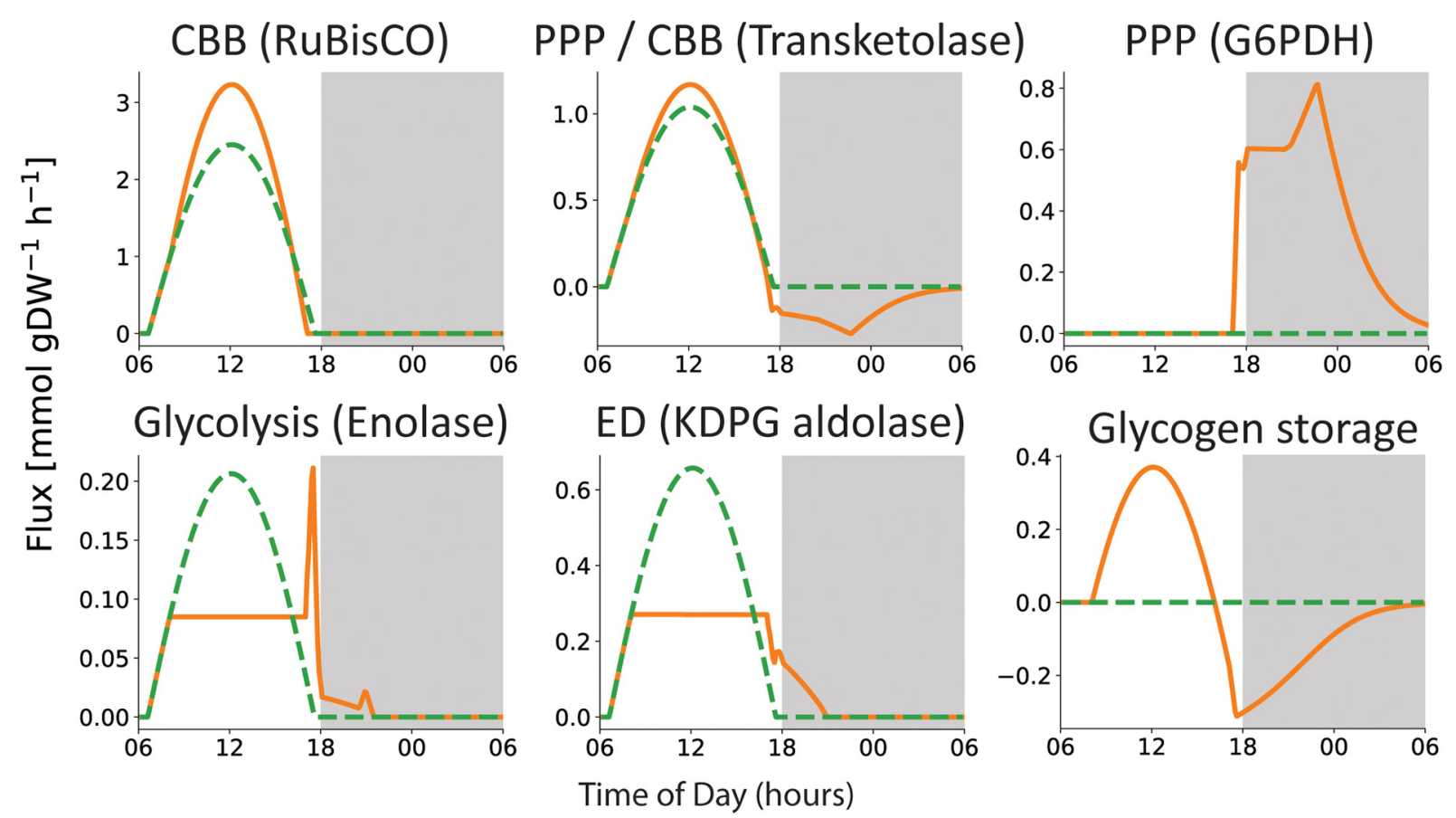

FIGURE 6 | Insight into metabolic rearrangements during the diel cycle. (A) Light irradiance, biomass and glycogen storage throughout the diel cycle. We observe that the largest accumulated growth is found in the nitrogen-abundant condition (green), but glycogen is only predicted to be stored in the nitrogen-poor condition (orange). (B) The flux distributions shifts when the metabolism switches from photosynthesis to glycogen catabolism, displayed by five reactions representing the Calvin Cycle (CBB), Glycogen metabolism, lower part of glycolysis, The Entner-Doudoroff (ED) and the Pentose Phosphate Pathway (PPP). (C) iSO595 predicts that the depletion of glycogen is accompanied by exudation of formate and pyruvate.

ATP demand necessary to support higher growth rates in nitrogen-abundant conditions. Additionally, our simulations predicted that the use of the Entner-Doudoroff pathway during photosynthesis creates precursor metabolites for growth during light hours, and a shift to the Pentose Phosphate Pathway (PPP) during nighttime. This trend might occur as an alternative for generating NADPH (Supplementary Figure 6). Upregulation of the PPP enzymes during dusk and the first half of the night time 
was also observed in the proteome of Prochlorococcus (Waldbauer et al., 2012). Several enzymatic transformations participate in both the Calvin cycle and the PPP, although in opposite directions (Waldbauer et al., 2012). These transformations were captured in our simulations, specifically as demonstrated by transketolase (Figure 6B). Additionally, the consumption of glycogen during nighttime might lead to exudation of pyruvate and formate (Figure 6C). This prediction is supported by recent observations; formate is exuded during both nutrient-replete and phosphatelimited growth in Prochlorococcus strains MED4 and MIT9312 under constant light (Bertilsson et al., 2005), as well as when phosphonates are metabolized in Prochlorococcus strain MIT9301 (Sosa et al., 2019). Thus, Prochlorococcus are potential formate sources for heterotrophs. However, degradation of phosphonates yields formate as an immediate byproduct, and the current modeling framework is not suited to evaluate whether a equally high amount of intracellular formate is feasible during glycogen degradation, as intracellular metabolite concentrations are not readily represented in $\mathrm{dFBA}$. Pyruvate exudation in Prochlorococcus is indicated from previous co-cultivations with SAR11 (Becker and Hogle, 2019), and from upregulation of genes encoding pyruvate kinase and a pyruvate efflux transporter during extended darkness (Biller et al., 2018). Furthermore, pyruvate is exuded when fixed carbon is consumed in the closely related strains S. elongatus PCC 7942 and S. sp. PCC 6803 (Carrieri et al., 2012; Benson et al., 2016).

The shift from photosynthesis and carbon fixation to glycogen catabolism is also associated with a switch in production and consumption of energetic cofactors (Supplementary Figure 6). Generation of ATP is performed concomitantly by ATP synthase in both the thylakoid membrane and the periplasmic membrane during photosynthesis. The periplasmic ATP synthase is first driven by reduced cofactors (NADPH) generated by the electron transport chain in the light-dependent part of photosynthesis (Supplementary Figure 6). ATP is consumed by two separate processes: growth- and maintenance-associated reactions reach a threshold once growth is limited by the nitrogen abundance, while the recycling of precursors for the Calvin cycle follows the shape of light absorption throughout the day. In agreement with previous work (Park and Choi, 2017), our model predicted higher rates of NADPH production than NADH.

Next, we explored the ability of our model to dynamically capture biologically relevant phenotypes by performing dynamic FBA simulations of knock-out mutants in Prochlorococcus, focusing on two gene deletions disrupting different parts of glycogen metabolism. $\triangle g l g C$ breaks synthesis of ADP-glucose and thus the storage of glycogen and $\Delta g n d$, knocking out 6-phosphogluconate dehydrogenase, a key reaction in the Pentose Phosphate pathway found to fuel the Calvin cycle with precursor metabolites during the onset of photosynthesis (Shinde et al., 2020). Our dynamic FBA simulations in COMETS (Supplementary Figure 7) showed similar growth between $\Delta$ gnd and the wild type and slightly lower growth for $\Delta g \operatorname{lgC}$. We set out to compare these observations with available experimental data. Since genetic tools for the modification of Prochlorococcus are still lacking (Laurenceau et al., 2020), we chose data from the closely related cyanobacteria Synechococcus as recent work described the impact of $\Delta g \operatorname{lgC}$ and $\Delta g n d$ on its growth during diel cycles (Shinde et al., 2020). Indeed, we found very good agreement between measured and predicted growth for both the wild-type and $\Delta g \lg C$ mutant where glycogen storage is disrupted (Shinde et al., 2020) (Supplementary Figure 7). One of the notable limitations of dynamic FBA is the ability to quantify intermediates and precursor pools that might drive the initiation of a pathway. This comes mainly from the assumption of a quasi steady-state of intracellular metabolite pools at each time point. Although the comparison is strictly qualitative and concerns strains with known differences (Mary et al., 2004), these findings demonstrated the ability of our reconstruction to capture metabolic trends in response to genetic perturbations, indicating that iSO595 will be a valuable tool in future research of Prochlorococcus. Overall, our dynamic simulations display biological and physiological behaviors that are consistent with expectations, and at the same time provide valuable insight into the putative internal metabolic processes that might modulate the Prochlorococcus growth under environmental and genomeinduced constraints.

\section{CONCLUSION}

Our study provides a detailed systematic view of the underlying metabolic trends modulating carbon storage and exudation in Prochlorococcus. Prochlorococcus is known to interact with other bacteria in its surroundings (Sher et al., 2011; Aharonovich and Sher, 2016; Biller et al., 2018; Hennon et al., 2018). It is currently impossible to predict the fluxes of organic matter (or of the myriad metabolites comprising it, such as amino acids, sugars, and organic acids) between phytoplankton and bacteria. Yet, quantifying such fluxes and predicting them from genomic surveys, as shown here, serves a number of roles: (1) It can provide experimentally testable and mechanistic hypotheses on inter-microbial exchanges and competition, (2) It has the potential to increase knowledge about the specific metabolites that may mediate these interactions; and (3) It would enable the construction of improved models of biogeochemical cycles which consider the diverse and powerful metabolic capabilities of the ocean microbiome.

Genome-scale metabolic-network reconstructions are powerful tools, but not without limitations. Mainly, the predictive accuracy rests on the quality and completeness of the metabolic network. The construction and curation of these metabolic networks depend heavily on data availability and annotation accuracy, which may be scarce for less studied organisms. Several methods have been developed to fill the gaps of incomplete network reconstructions. For example, FastGapFill incorporates missing knowledge from universal, non-organism specific data (Thiele et al., 2014), ModelSEED fills gaps through the use of thermodynamic parameters and FBA simulations to achieve minimal growth (Henry et al., 2010), and MENECO uses a topology graph based approach to look for minimal sets of metabolic reactions that support growth and the producibility of target metabolites (Prigent et al., 2017). In this work we used a novel semi-automated gap-filling method (ReFill) to 
increase existing knowledge in the reconstruction by up to $25 \%$. In contrast to other standard gap filling approaches, ReFill has the specific capability to add individual reactions through a recursive algorithm that guarantees complete connectivity to the existing network, incorporating the maximal possible amount of validated, organism specific metabolic annotations. However, this approach employs high stringency and thus adds limited amounts of knowledge. Considering that Prochlorococcus strains have some of the smallest known genomes among free-living organisms, a $25 \%$ increase in knowledge serves as a significant improvement in the predictive capacity of the model. However, reconstruction of high-quality genome-scale metabolic models is an iterative process, where new data, knowledge, and scope create opportunities for further model improvement. One example of this possibility is the $\mathrm{CO}_{2}$ concentrating mechanisms in Prochlorococcus. This mechanism is known to be sustained by proton and ion gradients across the cell membrane at an energetic cost (Hopkinson et al., 2014; Burnap et al., 2015). However, the comprehensive knowledge and annotation of ion transporters necessary to model this mechanism are lacking, and are therefore not included in iSO595. With the advancement of data collection and annotation tools, together with the use of ReFill or similar algorithms, metabolic knowledge can be added to such reconstructions, improving their predictive abilities and mimicry of biological and physiological processes.

Other limitations of static and dynamic FBA simulations include the inability to represent metabolite concentrations and the lack of regulatory effects. Furthermore, since COMETS, like most other implementations of $\mathrm{dFBA}$, simulates millions of asynchronously growing and dividing cells on the mesoscopic scale, cell cycle processes are not readily incorporated into this framework. Thus, future extensions to this work include the implementation of cell division in Prochlorococcus, known to occur in the afternoon (Vaulot et al., 1995). Another improvement would be an accurate representation of the costs associated with light damage and the production of protective pigments required to combat excessive light absorption. This could potentially be accounted for by extending the current Prochlorococcus GEM to a framework that includes macromolecular allocation, such as Resource Balance Analysis (Goelzer et al., 2011), conditional FBA (Rügen et al., 2015) or models of metabolism and macromolecular expression (ME models) (Thiele et al., 2012). Along these lines, one could relate mortality with an inability to maintain basic cellular functions, rather than a fixed death rate. However, the relationship between cell mortality and metabolism is not well constrained, and its representation in dFBA models is currently rudimentary. Future work is needed to better understand mortality and represent it in models of cell metabolism, ecosystems and biogeochemistry. Finally, our findings contribute to a growing body of work on the underlying metabolic mechanisms modulating the metabolic success of Prochlorococcus. The approaches shown here provide systematic insights corroborated in recent and well-known works and provide strong foundations for future studies of Prochlorococcus metabolism with particular interest in its interaction with other microorganisms and the effects of these on community composition and larger biogeochemical cycles.

\section{DATA AVAILABILITY STATEMENT}

Publicly available datasets were analyzed in this study. This data can be found here: KEGG (through Python API): https://www.genome.jp/kegg/, TransportDB: http: //www.membranetransport.org/transportDB2/index.html,

Metabolights: https://www.ebi.ac.uk/metabolights/MTBLS567, and former Prochlorococcus model: https://msystems.asm.org/ content/1/6/e00065-16.

\section{AUTHOR CONTRIBUTIONS}

DSh and DSe designed the study. SO, SS, and DSe developed the computational models and performed the computational analyses, with input from DSh. SO and SS wrote a first version of the manuscript. DSe, DSh, and EA oversaw the project and contributed to the final version of this manuscript. All authors have read and approved the final version of the manuscript.

\section{FUNDING}

This work was supported by the Human Frontiers Science Program (grant RGP0020/2016) and the National Science Foundation (NSFOCE-BSF 1635070) to DSe and DSh. DSe also acknowledges support by the National Science Foundation (grant 1457695), the Directorates for Biological Sciences and Geosciences at the National Science Foundation and NASA (agreement nos. 80NSSC17K0295, 80NSSC17K0296 and 1724150) issued through the Astrobiology Program of the Science Mission Directorate, and the Boston University Interdisciplinary Biomedical Research Office. SS was funded by SINTEF, the Norwegian graduate research school in bioinformatics, biostatistics and systems biology (NORBIS) and by the INBioPharm project of the Centre for Digital Life Norway (Research Council of Norway grant no. 248885).

\section{ACKNOWLEDGMENTS}

We are grateful to members of the Segrè lab for constructive feedback on the manuscript, and to members of the labs of DS, Hans-Peter Grossart, and Maren Voss for helpful and fun discussions on marine microbes.

\section{SUPPLEMENTARY MATERIAL}

The Supplementary Material for this article can be found online at: https://www.frontiersin.org/articles/10.3389/fgene. 2021.586293/full\#supplementary-material

Supplementary Figure 1 | Estimation of kinetic parameters for the uptake of ammonium in Prochlorococcus. (A) All combinations of $K_{m}$ and $V_{\max }$ along the red trajectory matches the observed gross growth rate of $0.5 \mathrm{~d}^{-1}$ (Grossowicz et al., 2017). However, when we compare the dynamics of cell density (B) and ammonium concentration (C) we find that the best overall prediction is achieved 
using $K_{\mathrm{m}}=0.39 \mathrm{mM}$ and $V_{\max }=0.9 \mathrm{mmol} \mathrm{gDW}^{-1} \mathrm{~h}^{-1}$ (marked by an orange dot in $\mathbf{A})$.

Supplementary Figure 2 | ATP availability influence modulates the trade-off between glycogen storage and growth. Phenotypic phase planes (Edwards et al., 2002) illustrate the combined effect of glycogen storage and bicarbonate uptake on the maximal growth rate. Compared to the base model $\mathbf{( A )}$, we observe how that the trade-off is strongly affected by modulated ATP availability, either from an artificial reaction providing extra ATP (B) or by increasing (C) or decreasing (D) the amount of available light. Increasing ATP allows more glycogen storage without reducing the growth rate.

Supplementary Figure 3 | TCA cycle flux diagram differences between the most common phenotypes. Flux diagrams of the TCA cycle in the most common phenotypes 2 (colored orange) and 3 (colored blue). Reactions are denoted by KEGG reaction ids. Reaction colors correspond to cluster colors presented in Figure 4.

Supplementary Figure 4 | Sensitivity analysis of dFBA simulations to variability in ammonium concentration, kinetic coefficients of ammonium uptake and light irradiance. (A) These phase diagrams display which combinations of $K_{m}$ and $V_{\text {max }}$, describing the uptake of ammonium, that leads to glycogen accumulation (red area) at peak irradiance based on the light amplitude and ammonium concentration used to simulate nitrogen-abundant (left) and nitrogen-poor (right) conditions in Figure 6. In the orange area no glycogen accumulation is predicted as the growth is limited by the available light, rather than nitrogen. (B) These panels display similar phase diagrams as in (A), but for different amplitudes of light irradiance, represented by black curves, and for different ammonium concentrations (indicated on top of each panel). The number written on each black curve represents the light irradiance amplitude in $\mu \mathrm{mol} Q$ $\mathrm{m}^{-2} \mathrm{~s}^{-1}$. The ammonium concentration in the top left panel is equal to the concentration in our simulated nitrogen-poor conditions, and thus, the 40.0 $40 \mu \mathrm{mol} \mathrm{Q} \mathrm{m} \mathrm{m}^{-2} \mathrm{~s}^{-1}$ line in this panel is identical to the boundary between the two phases in the right panel in (A). The range of ammonium concentrations is chosen so that it covers both our simulated environment and the ammonium concentration in oligotrophic oceans. The blue, green, and orange points display the combinations of $K_{\mathrm{m}}$ and $V_{\max }$ used/provided in this work and previous publications, respectively.

Supplementary Figure $\mathbf{5}$ | Sensitivity analysis of dFBA simulation to different parametrization of glycogen consumption. All panels display results obtained from dFBA simulations in COMETS with different combinations of $K_{m}$ and $V_{\max }$, describing the consumption of intracellular glycogen, with line colors and corresponding values as shown in the bottom right corner color matrix. The purple color corresponds to the values used to run the simulations shown in Figure 6 (A) Growth curves. (B) Accumulated glycogen per gram dry weight of biomass. (C) Predicted reaction fluxes for the same 8 reactions as shown in Figure 6.

\section{REFERENCES}

Aharonovich, D., and Sher, D. (2016). Transcriptional response of Prochlorococcus to co-culture with a marine Alteromonas: differences between strains and the involvement of putative infochemicals. ISME J. 10, 2892-2906. doi: 10.1038/ ismej.2016.70

Alonso-Casajús, N., Dauvillée, D., Viale, A. M., Muñoz, F. J., Baroja-Fernández, E., Morán-Zorzano, M. T., et al. (2006). Glycogen phosphorylase, the product of the glgP gene, catalyzes glycogen breakdown by removing glucose units from the nonreducing ends in Escherichia coli. J. Bacteriol. 188, 5266-5272. doi: 10.1128/JB.01566-05

Altschul, S., Gish, W., Miller, W., Myers, E., and Lipman, D. (1990). Basic local alignment search tool. J. Mol. Biol. 215, 403-410. doi: 10.1016/S0022-2836(05) 80360- 2

Amin, S. A., Parker, M. S., and Armbrust, E. V. (2012). Interactions between diatoms and bacteria. Microbiol. Mol. Biol. Rev. 76, 667-684. doi: 10.1128/ MMBR.00007-12

Arrieta, J. M., Mayol, E., Hansman, R. L., and Herndl, G. J. (2015). Dilution limits dissolved organic carbon utilization in the deep ocean. Science 348, $331-334$.
Supplementary Figure 6 | The transition from daytime to nighttime is associated with a drastic change in the production and consumption of the energy-carrying cofactors. The figure panels show the major sources (left) and drains (right) of the cofactors ATP, NAPDH and NADH. The legend shows the reaction IDs used in iSO595. (A) ATP is produced by both the thylakoid (R00086th) and the periplasmic (R00086p) ATP synthase during daytime, but mostly by the periplasmic ATP synthase (respiration) during nighttime. ATP is consumed by reactions associated with growth (BIOMASS and BProtein), cellular maintenance (Maintenance) and storage of glycogen (R00948) in addition to reactions recycling precursors for the Calvin cycle (R01512 and R01523) and acetyl-CoA carboxylase (R00742). (B) NADPH is produced by ferredoxin reductase (fdr) during daytime and by the pentose phosphate pathway (R02736 and R01528) during nighttime. The NADPH is either used to drive the proton gradient across the periplasmic membrane (NADPHDHp) or in Gluconeogenesis (R01063) to refuel the Calvin cycle during photosynthesis. (C) NADH production is correlated with the growth rate and dominated by pyruvate dehydrogenase (R00209) during daytime and the glycine cleavage system (R01221) during nighttime. NADH is consumed by 6-phosphogluconate dehydrogenase (reverse, R10221) during daytime, NADH transhydrogenase (R00112) solely during dusk and concomitant with methylenetetrahydrofolate reductase (R07168) during nighttime.

Supplementary Figure 7 | Predicted growth curves show good agreement in a qualitative comparison with experimental growth of Synechococcus. To compare growth data we have overlaid growth curves predicted for the wild-type, the $\Delta g / g C$-mutant and the $\Delta g n d$-mutant of Prochlorococcus with experimental OD measurements of Synechococcus elongatus PCC 794 (Shinde et al., 2020). We find a very good agreement for the wild-type and $\Delta g / g C$-mutant, but not for the $\Delta$ gnd-mutant. The lower panel shows how the model predicts the allocation and consumption of each of the three strains.

Supplementary Table 1 | List of reactions added to iJC568 to form iSO595.

Supplementary Table 2 | List of reactions from iJC568 that are modified in iSO595.

Supplementary Table 3 | Parameter ranges used in the sampling of nutrient environments.

Supplementary Table $\mathbf{4}$ | List of blocked exchange reactions prior to sampling of nutrient environments.

Supplementary Table 5 | Parameter values used to run dynamic FBA in COMETS.

Supplementary Material 1 | Results from the BLAST-search used to identify 6PG-dehydratase (EC: 4.2.1.12) encoded by PMM0774 in P. marinus MED4.

Supplementary Material 2 | Memote snapshot report of iSO595.

Arteaga, L., Pahlow, M., and Oschlies, A. (2016). Modeled Chl:C ratio and derived estimates of phytoplankton carbon biomass and its contribution to total particulate organic carbon in the global surface ocean. Global Biogeochem. Cycles 30, 1791-1810. doi: 10.1002/2016GB005458

Becker, J. W., Berube, P. M., Follett, C. L., Waterbury, J. B., Chisholm, S. W., Delong, E. F., et al. (2014). Closely related phytoplankton species produce similar suites of dissolved organic matter. Front. Microbiol. 5:111. doi: 10.3389/ fmicb.2014.00111

Becker, J. W., and Hogle, S. L. (2019). Co-culture and biogeography of Prochlorococcus and SAR11. ISME J. 13, 1506-1519. doi: 10.1038/s41396-0190365-4

Benson, P. J., Purcell-Meyerink, D., Hocart, C. H., Truong, T. T., James, G. O., Rourke, L., et al. (2016). Factors altering pyruvate excretion in a glycogen storage mutant of the cyanobacterium, synechococcus PCC7942. Front. Microbiol. 7:475. doi: 10.3389/fmicb.2016.00475

Bertilsson, S., Berglund, O., Pullin, M. J., and Chisholm, S. W. (2005). Release of dissolved organic matter by Prochlorococcus. Vie et Milieu 55, 225-231.

Biller, S. J., Berube, P. M., Lindell, D., and Chisholm, S. W. (2015). Prochlorococcus: the structure and function of collective diversity. Nat. Rev. Microbiol. 13, 13-27. doi: $10.1038 /$ nrmicro3378 
Biller, S. J., Coe, A., Roggensack, S. E., and Chisholm, W. (2018). Heterotroph interactions alter prochlorococcus transcriptome dynamics during extended periods of darkness. mSystems 3:e00040-18.

Braakman, R. (2019). Evolution of cellular metabolism and the rise of a globally productive biosphere. Free Radic. Biol. Med. 140, 172-187. doi: 10.1016/j. freeradbiomed.2019.05.004

Braakman, R., Follows, M. J., and Chisholm, S. W. (2017). Metabolic evolution and the self-organization of ecosystems. Proc. Natl. Acad. Sci. U.S.A. 114, E3091-E3100. doi: 10.1073/pnas.1619573114

Bricaud, A., Claustre, H., Ras, J., and Oubelkheir, K. (2004). Natural variability of phytoplanktonic absorption in oceanic waters: influence of the size structure of algal populations. J. Geophys. Res. Ocean 109, 1-12. doi: 10.1029/2004JC0 02419

Broddrick, J. T., Rubin, B. E., Welkie, D. G., Du, N., Mih, N., Diamond, S., et al. (2016). Unique attributes of cyanobacterial metabolism revealed by improved genome-scale metabolic modeling and essential gene analysis. Proc. Natl. Acad. Sci. U.S.A. 113, E8344-E8353. doi: 10.1073/pnas.1613446 113

Burnap, R., Hagemann, M., and Kaplan, A. (2015). Regulation of CO2 concentrating mechanism in cyanobacteria. Life 5, 348-371. doi: 10.3390/ life5010348

Campillo-Brocal, J. C., Lucas-Elió, P., and Sanchez-Amat, A. (2015). Distribution in different organisms of amino acid oxidases with fad or a quinone as cofactor and their role as antimicrobial proteins in marine bacteria. Mar. Drugs 13, 7403-7418. doi: $10.3390 / \mathrm{md} 13127073$

Cano, M., Holland, S. C., Artier, J., Burnap, R. L., Ghirardi, M., Morgan, J. A., et al. (2018). Glycogen synthesis and metabolite overflow contribute to energy balancing in cyanobacteria. Cell Rep. 23, 667-672. doi: 10.1016/j.celrep.2018.03. 083

Carrieri, D., Paddock, T., Maness, P.-C., Seibert, M., and Yu, J. (2012). Photocatalytic conversion of carbon dioxide to organic acids by a recombinant cyanobacterium incapable of glycogen storage. Energy Environ. Sci. 5:9457. doi: 10.1039/c2ee23181f

Casey, J. R., Mardinoglu, A., Nielsen, J., and Karl, D. M. (2016). Adaptive evolution of phosphorus metabolism in prochlorococcus. mSystems 1, 1-15. doi: 10.1128/ mSystems.00065-16.Editor

Chen, X., Schreiber, K., Appel, J., Makowka, A., Fähnrich, B., Roettger, M., et al. (2016). The Entner-Doudoroff pathway is an overlooked glycolytic route in cyanobacteria and plants. Proc. Natl. Acad. Sci. U.S.A. 113, 5441-5446. doi: 10.1073/pnas.1521916113

Cirri, E., and Pohnert, G. (2019). Algae-bacteria interactions that balance the planktonic microbiome. New Phytol. 223, 100-106. doi: 10.1111/nph.15765

Coles, V. J., Stukel, M. R., Brooks, M. T., Burd, A., Crump, B. C., Moran, M. A., et al. (2017). Ocean biogeochemistry modeled with emergent trait-based genomics. Science 358, 1149-1154. doi: 10.1126/science.aan5712

Damrow, R., Maldener, I., and Zilliges, Y. (2016). The multiple functions of common microbial carbon polymers, glycogen and PHB, during stress responses in the non-diazotrophic cyanobacterium synechocystis sp. PCC 6803. Front. Microbiol. 7:966. doi: 10.3389/fmicb.2016.00966

Dauvillée, D., Kinderf, I. S., Li, Z., Kosar-Hashemi, B., Samuel, M. S., Rampling, L., et al. (2005). Role of the Escherichia coli glgX gene in glycogen metabolism. J. Bacteriol. 187, 1465-1473. doi: 10.1128/JB.187.4.1465-1473.2005

Davey, M., Tarran, G. A., Mills, M. M., Ridame, C., Geider, R. J., and LaRoche, J. (2008). Nutrient limitation of picophytoplankton photosynthesis and growth in the tropical North Atlantic. Limnol. Oceanogr. 53, 1722-1733. doi: 10.4319/lo. 2008.53.5.1722

de Groot, D. H., Lischke, J., Muolo, R., Planqué, R., Bruggeman, F. J., and Teusink, B. (2020). The common message of constraint-based optimization approaches: overflow metabolism is caused by two growth-limiting constraints. Cell. Mol. Life Sci. 77, 441-453. doi: 10.1007/s00018-019-03380-2

Deutsch, C., Sarmiento, J. L., Sigman, D. M., Gruber, N., and Dunne, J. P. (2007). Spatial coupling of nitrogen inputs and losses in the ocean. Nature 445, 163-167. doi: 10.1038/nature05392

Diamond, S., Jun, D., Rubin, B. E., and Golden, S. S. (2015). The circadian oscillator in Synechococcus elongatus controls metabolite partitioning during diurnal growth. Proc. Natl. Acad. Sci. U.S.A. 112, E1916-E1925. doi: 10.1073/pnas. 1504576112
Díaz-Troya, S., López-Maury, L., Sánchez-Riego, A. M., Roldán, M., and Florencio, F. J. (2014). Redox regulation of glycogen biosynthesis in the cyanobacterium synechocystis sp. PCC 6803: analysis of the AGP and glycogen synthases. Mol. Plant 7, 87-100. doi: 10.1093/mp/sst137

Domínguez-Martín, M. A., López-Lozano, A., Clavería-Gimeno, R., VelázquezCampoy, A., Seidel, G., Burkovski, A., et al. (2018). Differential NtcA responsiveness to 2-oxoglutarate underlies the diversity of $\mathrm{C} / \mathrm{N}$ balance regulation in Prochlorococcus. Front. Microbiol. 8:2641. doi: 10.3389/fmicb. 2017.02641

Dubinsky, Z., and Berman-Frank, I. (2001). Uncoupling primary production from population growth in photosynthesizing organisms in aquatic ecosystems. Aquatic Sci. 63, 4-17. doi: 10.1007/PL00001343

Dukovski, I., Bajić, D., Chacón, J. M., Quintin, M., Vila, J. C., Sulheim, S., et al. (2020). ). Computation of Microbial Ecosystems in Time and Space (COMETS): An Open Source Collaborative Platform for Modeling Ecosystems Metabolism. Available online at: http://arxiv.org/abs/2009.01734 (accessed December 17, 2020).

Ebrahim, A., Lerman, J. A., Palsson, B. O., and Hyduke, D. R. (2013). COBRApy: constraints-based reconstruction and analysis for python. BMC Syst. Biol. 7:74. doi: 10.1186/1752-0509-7-74

Edwards, J. S., Ramakrishna, R., and Palsson, B. O. (2002). Characterizing the metabolic phenotype: a phenotype phase plane analysis. Biotechnol. Bioeng. 77, 27-36. doi: 10.1002/bit.10047

Elbourne, L. D. H., Tetu, S. G., Hassan, K. A., and Paulsen, I. T. (2017). TransportDB 2.0: a database for exploring membrane transporters in sequenced genomes from all domains of life. Nucleic Acids Res. 45, D320-D324. doi: 10.1093/nar/gkw1068

Feist, A. M., Nagarajan, H., Rotaru, A. E., Tremblay, P. L., Zhang, T., Nevin, K. P., et al. (2014). Constraint-based modeling of carbon fixation and the energetics of electron transfer in geobacter metallireducens. PLoS Comput. Biol. 10:e1003575. doi: 10.1371/journal.pcbi.1003575

Field, C. B., Behrenfeld, M. J., Randerson, J. T., and Falkowski, P. (1998). Primary production of the biosphere: integrating terrestrial and oceanic components. Science 281, 237-240. doi: 10.1126/science.281.5374.237

Fischer, E., and Sauer, U. (2005). Large-scale in vivo flux analysis shows rigidity and suboptimal performance of Bacillus subtilis metabolism. Nat. Genet. 37, 636-640. doi: 10.1038/ng1555

Flombaum, P., Gallegos, J. L., Gordillo, R. A., Rincón, J., Zabala, L. L., Jiao, N., et al. (2013). Present and future global distributions of the marine Cyanobacteria Prochlorococcus and Synechococcus. Proc. Natl. Acad. Sci. U.S.A. 110, 98249829. doi: 10.1073/pnas.1307701110

Fogg, G. E., Nalewajko, C., and Watt, W. D. (1965). Extracellular products of phytoplankton photosynthesis. Proc. R. Soc. London. Ser. B. Biol. Sci. 162, 517-534. doi: 10.1098/rspb.1965.0054

Follows, M. J., Dutkiewicz, S., Grant, S., and Chisholm, S. W. (2007). Emergent biogeography of microbial communities in a model ocean. Science 315, 18431846. doi: $10.1126 /$ science. 1138544

Forchhammer, K., and Schwarz, R. (2019). Nitrogen chlorosis in unicellular cyanobacteria - a developmental program for surviving nitrogen deprivation. Environ. Microbiol. 21, 1173-1184. doi: 10.1111/1462-2920.14447

Forchhammer, K., and Selim, K. A. (2019). Carbon/nitrogen homeostasis control in cyanobacteria. FEMS Microbiol. Rev. 44, 33-53. doi: 10.1093/femsre/fuz025

Foster, S. Q., Al-haj, A., Church, M. J., van Dijken, G. L., Dutkiewicz, S., Fulweiler, R. W., et al. (2018). Ecological control of nitrite in the upper ocean. Nat. Commun. 9:1206. doi: 10.1038/s41467-018-03553-w

$\mathrm{Fu}, \mathrm{J}$., and $\mathrm{Xu}, \mathrm{X}$. (2006). The functional divergence of two glgP homologues in Synechocystis sp. PCC 6803. FEMS Microbiol. Lett. 260, 201-209. doi: 10.1111/ j.1574-6968.2006.00312.x

Gilbert, J. D. J., and Fagan, W. F. (2011). Contrasting mechanisms of proteomic nitrogen thrift in Prochlorococcus. Mol. Ecol. 20, 92-104. doi: 10.1111/j.1365294X.2010.04914.x

Goelzer, A., Fromion, V., and Scorletti, G. (2011). Cell design in bacteria as a convex optimization problem. Automatica 47, 1210-1218. doi: 10.1016/j. automatica.2011.02.038

Gomez, J. A., Höffner, K., and Barton, P. I. (2014). DFBAlab: a fast and reliable MATLAB code for dynamic flux balance analysis. BMC Bioinform. 15:409. doi: 10.1186/s12859-014-0409-8 
Grossowicz, M., Roth-Rosenberg, D., Aharonovich, D., Silverman, J., Follows, M. J., and Sher, D. (2017). Prochlorococcus in the lab and in silico: the importance of representing exudation. Limnol. Oceanogr. 62, 818-835. doi: 10.1002/lno.10463

Gu, C., Kim, G. B., Kim, W. J., Kim, H. U., and Lee, S. Y. (2019). Current status and applications of genome-scale metabolic models. Genome Biol. 20, 1-18. doi: 10.1186/s13059-019-1730-3

Gudmundsson, S., and Thiele, I. (2010). Computationally efficient flux variability analysis. BMC Bioinform. 11:489. doi: 10.1186/1471-2105-11-489

Harcombe, W. R., Riehl, W. J., Dukovski, I., Granger, B. R., Betts, A., Lang, A. H., et al. (2014). Metabolic resource allocation in individual microbes determines ecosystem interactions and spatial dynamics. Cell Rep. 7, 1104-1115. doi: 10. 1016/j.celrep.2014.03.070

Haug, K., Salek, R. M., Conesa, P., Hastings, J., De Matos, P., Rijnbeek, M., et al. (2013). MetaboLights-an open-access general-purpose repository for metabolomics studies and associated meta-data. Nucleic Acids Res. 41, 781-786. doi: 10.1093/nar/gks1004

Heirendt, L., Arreckx, S., Pfau, T., Mendoza, S. N., Richelle, A., Heinken, A., et al. (2019). Creation and analysis of biochemical constraint-based models using the COBRA Toolbox v.3.0. Nat. Protoc. 14, 639-702. doi: 10.1038/s41596-0180098-2

Hennon, G. M., Morris, J. J., Haley, S. T., Zinser, E. R., Durrant, A. R., Entwistle, E., et al. (2018). The impact of elevated CO 2 on Prochlorococcus and microbial interactions with â $€$ helper' bacterium Alteromonas. ISME J. 12, 520-531. doi: 10.1038/ismej.2017.189

Henry, C. S., Dejongh, M., Best, A. A., Frybarger, P. M., Linsay, B., and Stevens, R. L. (2010). High-throughput generation, optimization and analysis of genomescale metabolic models. Nat. Biotechnol. 28, 977-982. doi: 10.1038/nbt.1672

Holtzendorff, J., Partensky, F., Mella, D., Lennon, J.-F., Hess, W. R., and Garczarek, L. (2008). Genome streamlining results in loss of robustness of the circadian clock in the marine cyanobacterium Prochlorococcus marinus PCC 9511. J. Biol. Rhythms 23, 187-199. doi: 10.1177/0748730408316040

Hopkinson, B. M., Young, J. N., Tansik, A. L., and Binder, B. J. (2014). The minimal $\mathrm{CO} 2$-concentrating mechanism of prochlorococcus spp. MED4 is effective and efficient. Plant Physiol. 166, 2205-2217. doi: 10.1104/pp.114.247049

Iglesias, A. A., Kakefuda, G., and Preiss, J. (1991). Regulatory and structural properties of the cyanobacterial ADPglucose pyrophosphorylases. Plant Physiol. 97, 1187-1195. doi: 10.1104/pp.97.3.1187

Johnson, Z. I., Zinser, E. R., Coe, A., McNulty, N. P., Woodward, E. M. S., and Chisholm, S. W. (2006). Niche partitioning among Prochlorococcus ecotypes along ocean-scale environmental gradients. Science 311, 1737-1740. doi: 10. $1126 /$ science. 1118052

Kanehisa, M., and Goto, S. (2000). KEGG: kyoto encyclopedia of genes and genomes. Nucleic Acids Res. 28, 27-30.

Kashyap, A. K., and Singh, D. P. (1985). Ammonium transport in unicellular cyanobacterium anacystis nidulans. J. Plant Physiol. 121, 319-330. doi: 10.1016/ S0176-1617(85)80025-0

Kavvas, E. S., Seif, Y., Yurkovich, J. T., Norsigian, C., Poudel, S., Greenwald, W. W., et al. (2018). Updated and standardized genome-scale reconstruction of Mycobacterium tuberculosis H37Rv, iEK1011, simulates flux states indicative of physiological conditions. BMC Syst. Biol. 12:25. doi: 10.1186/s12918-018-0557$\mathrm{y}$

Kettler, G. C., Martiny, A. C., Huang, K., Zucker, J., Coleman, M. L., Rodrigue, S., et al. (2007). Patterns and implications of gene gain and loss in the evolution of prochlorococcus. PLoS Genet. 3:e231. doi: 10.1371/journal.pgen.0030231

Kim, J., Fabris, M., Baart, G., Kim, M. K., Goossens, A., Vyverman, W., et al. (2016). Flux balance analysis of primary metabolism in the diatom Phaeodactylum tricornutum. Plant J. 85, 161-176. doi: 10.1111/tpj.13081

Knoop, H., Gründel, M., Zilliges, Y., Lehmann, R., Hoffmann, S., Lockau, W., et al. (2013). Flux balance analysis of cyanobacterial metabolism: the metabolic network of Synechocystis sp. PCC 6803. PLoS Comput. Biol. 9:e1003081. doi: 10.1371/journal.pcbi.1003081

Krumhardt, K. M., Callnan, K., Roache-Johnson, K., Swett, T., Robinson, D., Reistetter, E. N., et al. (2013). Effects of phosphorus starvation versus limitation on the marine cyanobacterium ProchlorococcusMED4 I: uptake physiology. Environ. Microbiol. 15, 2114-2128. doi: 10.1111/1462-2920.12079

Kujawinski, E. B. (2011). The impact of microbial metabolism on marine dissolved organic matter. Ann. Rev. Mar. Sci. 3, 567-599. doi: 10.1146/annurev-marine120308-081003
Laurenceau, R., Bliem, C., Osburne, M. S., Becker, J. W., Biller, S. J., CubillosRuiz, A., et al. (2020). Toward a genetic system in the marine cyanobacterium Prochlorococcus. Access Microbiol. 2:e000107. doi: 10.1099/acmi.0.000107

Lewis, N. E., Hixson, K. K., Conrad, T. M., Lerman, J. A., Charusanti, P., Polpitiya, A. D., et al. (2010). Omic data from evolved E. coli are consistent with computed optimal growth from genome-scale models. Mol. Syst. Biol. 6:390. doi: 10.1038/ msb. 2010.47

Lieven, C., Beber, M. E., Olivier, B. G., Bergmann, F. T., Ataman, M., Babaei, P., et al. (2020). MEMOTE for standardized genome-scale metabolic model testing. Nat. Biotechnol. 38, 272-276. doi: 10.1038/s41587-020-0446-y

Long, S. P., Humphries, S., and Falkowski, P. G. (1994). Photoinhibition of photosynthesis in nature. Annu. Rev. Plant Physiol. Plant Mol. Biol. 45, 633-662. doi: 10.1146/annurev.pp.45.060194.003221

López-Sandoval, D. C., Rodríguez-Ramos, T., Cermeño, P., and Marañón, E. (2013). Exudation of organic carbon by marine phytoplankton: dependence on taxon and cell size. Mar. Ecol. Prog. Ser. 477, 53-60. doi: 10.3354/meps1 0174

Luan, G., Zhang, S., Wang, M., and Lu, X. (2019). Progress and perspective on cyanobacterial glycogen metabolism engineering. Biotechnol. Adv. 37, 771-786. doi: 10.1016/j.biotechadv.2019.04.005

Ma, L., Calfee, B. C., Morris, J. J., Johnson, Z. I., and Zinser, E. R. (2018). Degradation of hydrogen peroxide at the ocean's surface: the influence of the microbial community on the realized thermal niche of Prochlorococcus. ISME J. 12, 473-484. doi: 10.1038/ismej.2017.182

Ma, X., Coleman, M. L., and Waldbauer, J. R. (2018). Distinct molecular signatures in dissolved organic matter produced by viral lysis of marine cyanobacteria. Environ. Microbiol. 20, 3001-3011. doi: 10.1111/1462-2920.14338

Maarleveld, T. R., Khandelwal, R. A., Olivier, B. G., Teusink, B., and Bruggeman, F. J. (2013). Basic concepts and principles of stoichiometric modeling of metabolic networks. Biotechnol. J. 8, 997-1008. doi: 10.1002/biot.201200291

Mague, T. H., Friberg, E., Hughes, D. J., and Morris, I. (1980). Extracellular release of carbon by marine phytoplankton; a physiological approach. Limnol. Oceanogr. 25, 262-279. doi: 10.4319/lo.1980.25.2.0262

Mahadevan, R., Edwards, J. S., and Doyle, F. J. (2002). Dynamic Flux Balance Analysis of diauxic growth in Escherichia coli. Biophys. J. 83, 1331-1340. doi: 10.1016/S0006-3495(02)73903-9

Marañón, E., Cermeño, P., López-Sandoval, D. C., Rodríguez-Ramos, T., Sobrino, C., Huete-Ortega, M., et al. (2013). Unimodal size scaling of phytoplankton growth and the size dependence of nutrient uptake and use. Ecol. Lett. 16, 371-379. doi: 10.1111/ele.12052

Mary, I., Tu, C.-J., Grossman, A., and Vaulot, D. (2004). Effects of high light on transcripts of stress-associated genes for the cyanobacteria Synechocystis sp. PCC 6803 and Prochlorococcus MED4 and MIT9313. Microbiology 150, 1271-1281. doi: 10.1099/mic.0.27014-0

McDonald, S. M., Plant, J. N., and Worden, A. Z. (2010). The mixed lineage nature of nitrogen transport and assimilation in marine eukaryotic phytoplankton: a case study of Micromonas. Mol. Biol. Evol. 27, 2268-2283. doi: 10.1093/molbev/ msq113

McInnes, L., Healy, J., and Astels, S. (2017). hdbscan: hierarchical density based clustering. J. Open Source Softw. 2:205. doi: 10.21105/joss.00205

McKinney, W. (2010). pandas: a Foundational Python Library for Data Analysis and Statistics $\mid R$ (Programming Language) | Database Index. Available online at: https://www.scribd.com/document/71048089/pandas-a-FoundationalPython-Library-for-Data-Analysis-and-Statistics (accessed April 29, 2020).

Mella-Flores, D., Six, C., Ratin, M., Partensky, F., Boutte, C., Le Corguillé, G., et al. (2012). Prochlorococcus and synechococcus have evolved different adaptive mechanisms to cope with light and UV Stress. Front. Microbiol. 3:285. doi: 10.3389/fmicb.2012.00285

Monk, J. M., Lloyd, C. J., Brunk, E., Mih, N., Sastry, A., King, Z., et al. (2017). iML1515, a knowledgebase that computes Escherichia coli traits. Nat. Biotechnol. 35, 904-908. doi: 10.1038/nbt.3956

Monshupanee, T., and Incharoensakdi, A. (2014). Enhanced accumulation of glycogen, lipids and polyhydroxybutyrate under optimal nutrients and light intensities in the cyanobacterium Synechocystis sp. PCC 6803. J. Appl. Microbiol. 116, 830-838. doi: 10.1111/jam.12409

Moore, C. M., Mills, M. M., Arrigo, K. R., Berman-Frank, I., Bopp, L., Boyd, P. W., et al. (2013). Processes and patterns of oceanic nutrient limitation. Nat. Geosci. 6, 701-710. doi: 10.1038/ngeo 1765 
Moore, L. R., Coe, A., Zinser, E. R., Saito, M. A., Sullivan, M. B., Lindell, D., et al. (2007). Culturing the marine cyanobacterium Prochlorococcus. Limnol. Oceanogr. Methods 5, 353-362. doi: 10.4319/lom.2007.5.353

Moore, L. R., Goericke, R., and Chisholm, S. W. (1995). Comparative physiology of Synechococcus and Prochlorococcus: influence of light and temperature on growth, pigments, fluorescence and absorptive properties. Mar. Ecol. Prog. Ser. $116,259-275$.

Moradi, N., Liu, B., Iversen, M., Kuypers, M. M., Ploug, H., and Khalili, A. (2018). A new mathematical model to explore microbial processes and their constraints in phytoplankton colonies and sinking marine aggregates. Sci. Adv. 4, 1-10. doi: 10.1126/sciadv.aat1991

Moran, M. A., and Durham, B. P. (2019). Sulfur metabolites in the pelagic ocean. Nat. Rev. Microbiol. 17, 665-678. doi: 10.1038/s41579-019-0250-1

Moran, M. A., Kujawinski, E. B., Stubbins, A., Fatland, R., Aluwihare, L. I., Buchan, A., et al. (2016). Deciphering ocean carbon in a changing world. Proc. Natl. Acad. Sci. U.S.A. 113, 3143-3151. doi: 10.1073/pnas.1514645113

Morel, A., and Bricaud, A. (1981). Theoretical results concerning light absorption in a discrete medium, and application to specific absorption of phytoplankton. Deep Sea Res. Part A Oceanogr. Res. Pap. 28, 1375-1393. doi: 10.1016/01980149(81)90039-X

Morris, J. J., Lenski, R. E., and Zinser, E. R. (2012). The black queen hypothesis: evolution of dependencies through adaptive gene loss. MBio 3:e00036-12. doi: 10.1128/mBio.00036-12

Muñoz-Marín, M. C., Gómez-Baena, G., López-Lozano, A., Moreno-Cabezuelo, J. A., Díez, J., and García-Fernández, J. M. (2020). Mixotrophy in marine picocyanobacteria: use of organic compounds by Prochlorococcus and Synechococcus. ISME J. 14, 1065-1073. doi: 10.1038/s41396-020-0 603-9

Nicholson, D. P., Stanley, R. H. R., and Doney, S. C. (2018). A Phytoplankton model for the allocation of gross photosynthetic energy including the tradeoffs of diazotrophy. J. Geophys. Res. Biogeosci. 123, 1796-1816. doi: 10.1029/ 2017JG004263

Nogales, J., Gudmundsson, S., Knight, E. M., Palsson, B. O., and Thiele, I. (2012). Detailing the optimality of photosynthesis in cyanobacteria through systems biology analysis. Proc. Natl. Acad. Sci. U.S.A. 109, 2678-2683. doi: 10.1073/pnas. 1117907109

Noreña-Caro, D., and Benton, M. G. (2018). Cyanobacteria as photoautotrophic biofactories of high-value chemicals. J. CO2 Util. 28, 335-366. doi: 10.1016/j. jcou.2018.10.008

O’Brien, E. J., Monk, J. M., and Palsson, B. O. (2015). Using genome-scale models to predict biological capabilities. Cell 161, 971-987. doi: 10.1016/j.cell.2015.05.019

Orth, J. D., Thiele, I., and Palsson, B. O. (2010). What is flux balance analysis? Nat. Biotechnol. 28, 245-248. doi: 10.1038/nbt.1614

Oschlies, A., Koeve, W., Landolfi, A., and Kähler, P. (2019). Loss of fixed nitrogen causes net oxygen gain in a warmer future ocean. Nat. Commun. 10, 1-7. doi: 10.1038/s41467-019-10813-w

Pacheco, A. R., Moel, M., and Segrè, D. (2018). Costless metabolic secretions as drivers of interspecies interactions in microbial ecosystems. Nat. Commun. 10:103.

Park, J., and Choi, Y. (2017). Cofactor engineering in cyanobacteria to overcome imbalance between NADPH and NADH: a mini review. Front. Chem. Sci. Eng. 11:66-71. doi: 10.1007/s11705-016-1591-1

Partensky, F., and Garczarek, L. (2010). Prochlorococcus: advantages and limits of minimalism. Ann. Rev. Mar. Sci. 2, 305-331. doi: 10.1146/annurev-marine120308-081034

Partensky, F., Hess, W. R., and Vaulot, D. (1999). Prochlorococcus, a marine photosynthetic prokaryote of global significance. Microbiol. Mol. Biol. Rev. 63, 106-127. doi: 10.1128/mmbr.63.1.106-127.1999

Pedregosa, F., Varoquaux, G., Gramfort, A., Michel, V., Thirion, B., Grisel, O., et al. (2011). Scikit-learn: machine learning in python. J. Mach. Learn. Res 12, 2825-2830.

Pope, R. M., and Fry, E. S. (1997). Absorption spectrum (380-700 nm) of pure water. II. Integrating cavity measurements. Appl. Optics 33, 8710-8723. doi: 10.1364/AO.36.008710

Prigent, S., Frioux, C., Dittami, S. M., Thiele, S., Larhlimi, A., Collet, G., et al. (2017). Meneco, a topology-based gap-filling tool applicable to degraded genome-wide metabolic networks. PLoS Comput. Biol. 13:e1005276. doi: 10. 1371/journal.pcbi.1005276
Reid, A. (2012). Incorporating Microbial Processes into Climate Change Models. A Report by the American Academy of Microbiology. Washington, DC.

Reimers, A.-M., Knoop, H., Bockmayr, A., and Steuer, R. (2017). Cellular tradeoffs and optimal resource allocation during cyanobacterial diurnal growth. Proc. Natl. Acad. Sci. U.S.A. 114:201617508. doi: 10.1073/pnas.1617508114

Ribalet, F., Swalwell, J., Clayton, S., Jiménez, V., Sudek, S., Lin, Y., et al. (2015). Light-driven synchrony of Prochlorococcus growth and mortality in the subtropical Pacific gyre. Proc. Natl. Acad. Sci. U.S.A. 112, 8008-8012. doi: 10. 1073/pnas.1424279112

Roth-rosenberg, D., Aharonovich, D., Omta, A.-W., Follows, M. J., and Sciences, P. (2019). Dynamic macromolecular composition and high exudation rates in Prochlorococcus. bioRxiv [Preprint]. doi: 10.1101/828897

Rügen, M., Bockmayr, A., and Steuer, R. (2015). Elucidating temporal resource allocation and diurnal dynamics in phototrophic metabolism using conditional FBA. Sci. Rep. 5, 1-16. doi: 10.1038/srep15247

Saito, M. A., McIlvin, M. R., Moran, D. M., Goepfert, T. J., DiTullio, G. R., Post, A. F., et al. (2014). Multiple nutrient stresses at intersecting Pacific Ocean biomes detected by protein biomarkers. Science 345, 1173-1177. doi: 10.1126/ science. 1256450

Schuetz, R., Zamboni, N., Zampieri, M., Heinemann, M., and Sauer, U. (2012). Multidimensional optimality of microbial metabolism. Science 336, 601-604. doi: $10.1126 /$ science. 1216882

Seaver, L. C., and Imlay, J. A. (2001). Hydrogen peroxide fluxes and compartmentalization inside growing Escherichia coli. J. Bacteriol. 183, 71827189. doi: 10.1128/JB.183.24.7182-7189.2001

Segrè, D., Vitkup, D., and Church, G. M. (2002). Analysis of optimality in natural and perturbed metabolic networks. Proc. Natl. Acad. Sci. U.S.A. 99, 1511215117. doi: 10.1073/pnas.232349399

Sher, D., Thompson, J. W., Kashtan, N., Croal, L., and Chisholm, S. W. (2011). Response of Prochlorococcus ecotypes to co-culture with diverse marine bacteria. ISME J. 5, 1125-1132. doi: 10.1038/ismej.2011.1

Shinde, S., Zhang, X., Singapuri, S. P., Kalra, I., Liu, X., Morgan-Kiss, R. M., et al. (2020). Glycogen metabolism supports photosynthesis start through the oxidative pentose phosphate pathway in cyanobacteria. Plant Physiol. 182, 507-517. doi: 10.1104/pp.19.01184

Sosa, O. A., Casey, J. R., and Karl, D. M. (2019). Methylphosphonate oxidation in Prochlorococcus strain MIT9301 supports phosphate acquisition, formate excretion, and carbon assimilation into purines. Appl. Environ. Microbiol. 85:e00289-19. doi: 10.1128/AEM.00289-19

Steglich, C., Behrenfeld, M., Koblizek, M., Claustre, H., Penno, S., Prasil, O., et al. (2001). Nitrogen deprivation strongly affects Photosystem II but not phycoerythrin level in the divinyl-chlorophyll b-containing cyanobacterium Prochlorococcus marinus. Biochim. Biophys. Acta Bioenerg. 1503, 341-349. doi: 10.1016/S0005-2728(00)00211-5

Stramski, D., Bricaud, A., and Morel, A. (2001). Modeling the inherent optical properties of the ocean based on the detailed composition of the planktonic community. Appl. Optics 18, 2929-2945. doi: 10.1364/ao.40.002929

Szul, M. J., Dearth, S. P., Campagna, S. R., and Zinser, E. R. (2019). Carbon fate and flux in prochlorococcus under nitrogen limitation. mSystems 4:e00254-18. doi: 10.1128/msystems.00254- 18

Thiele, I., Fleming, R. M. T., Que, R., Bordbar, A., Diep, D., and Palsson, B. O. (2012). Multiscale modeling of metabolism and macromolecular synthesis in E. coli and its application to the evolution of codon usage. PLoS One 7:e45635. doi: 10.1371/journal.pone.0045635

Thiele, I., Hyduke, D. R., Steeb, B., Fankam, G., Allen, D. K., Bazzani, S., et al. (2011). A community effort towards a knowledge-base and mathematical model of the human pathogen Salmonella Typhimurium LT2. BMC Syst. Biol. 5:8. doi: 10.1186/1752-0509-5-8

Thiele, I., Vlassis, N., and Fleming, R. M. T. (2014). FASTGAPFILL: efficient gap filling in metabolic networks. Bioinformatics 30, 2529-2531. doi: 10.1093/ bioinformatics/btu321

Thornton, D. C. O. (2014). Dissolved organic matter (DOM) release by phytoplankton in the contemporary and future ocean. Eur. J. Phycol. 49, 20-46. doi: 10.1080/09670262.2013.875596

van der Maaten, L., and Hinton, G. (2008). Visualizing data using t-SNE. J. Mach. Learn. Res. 9, 2579-2605.

Van Mooy, B. A. S., Rocap, G., Fredricks, H. F., Evans, C. T., and Devol, A. H. (2006). Sulfolipids dramatically decrease phosphorus demand by 
picocyanobacteria in oligotrophic marine environments. Proc. Natl. Acad. Sci. U.S.A. 103, 8607-8612. doi: 10.1073/pnas.0600540103

Varma, A., and Palsson, B. O. (1994). Metabolic flux balancing: basic concepts, scientific and practical use. Bio/Technology 12, 994-998. doi: 10.1038/nbt10 94-994

Vaulot, D., Marie, D., Olson, R. J., and Chisholm, S. W. (1995). Growth of Prochlorococcus, a photosynthetic prokaryote, in the equatorial Pacific Ocean. Science 268, 1480-1482. doi: 10.1126/science.268.5216.1480

Virtanen, P., Gommers, R., Oliphant, T. E., Haberland, M., Reddy, T., Cournapeau, D., et al. (2020). SciPy 1.0: fundamental algorithms for scientific computing in Python. Nat. Methods 17, 261-272. doi: 10.1038/s41592-019-0 $686-2$

Waldbauer, J. R., Rodrigue, S., Coleman, M. L., and Chisholm, S. W. (2012). Transcriptome and proteome dynamics of a light-dark synchronized bacterial cell cycle. PLoS One 7:e43432. doi: 10.1371/journal.pone.0043432

Ward, B. A., Collins, S., Dutkiewicz, S., Gibbs, S., Bown, P., Ridgwell, A., et al. (2019). Considering the role of adaptive evolution in models of the ocean and climate system. J. Adv. Model. Earth Syst. 11, 3343-3361. doi: 10.1029/ 2018MS001452

Welkie, D. G., Rubin, B. E., Diamond, S., Hood, R. D., Savage, D. F., and Golden, S. S. (2019). A hard day's night: cyanobacteria in diel cycles. Trends Microbiol. 27, 231-242.

Wintermute, E. H., Lieberman, T. D., and Silver, P. A. (2013). An objective function exploiting suboptimal solutions in metabolic networks. BMC Syst. Biol. 7:98. doi: 10.1186/1752-0509-7-98

Xiong, W., Cano, M., Wang, B., Douchi, D., and Yu, J. (2017). The plasticity of cyanobacterial carbon metabolism. Curr. Opin. Chem. Biol. 41, 12-19. doi: 10.1016/j.cbpa.2017.09.004
Yang, A. (2011). Modeling and evaluation of CO 2 supply and utilization in algal ponds. Ind. Eng. Chem. Res. 50, 11181-11192. doi: 10.1021/ie200 $723 \mathrm{w}$

Yoshikawa, K., Toya, Y., and Shimizu, H. (2017). Metabolic engineering of Synechocystis sp. PCC 6803 for enhanced ethanol production based on flux balance analysis. Bioprocess Biosyst. Eng. 40, 791-796. doi: 10.1007/s00449-0171744-8

Zavřel, T., Faizi, M., Loureiro, C., Poschmann, G., Stühler, K., Sinetova, M., et al. (2019). Quantitative insights into the cyanobacterial cell economy. eLife 8:e42508. doi: 10.7554/eLife.42508

Zhang, C. C., Zhou, C. Z., Burnap, R. L., and Peng, L. (2018). Carbon/nitrogen metabolic balance: lessons from cyanobacteria. Trends Plant Sci. 23, 1116-1130. doi: 10.1016/j.tplants.2018.09.008

Zinser, E. R., Lindell, D., Johnson, Z. I., Futschik, M. E., Steglich, C., Coleman, M. L., et al. (2009). Choreography of the transcriptome, photophysiology, and cell cycle of a minimal photoautotroph, Prochlorococcus. PLoS One 4:e5135. doi: 10.1371/journal.pone.0005135

Conflict of Interest: The authors declare that the research was conducted in the absence of any commercial or financial relationships that could be construed as a potential conflict of interest.

Copyright (c) 2021 Ofaim, Sulheim, Almaas, Sher and Segrè. This is an open-access article distributed under the terms of the Creative Commons Attribution License (CC BY). The use, distribution or reproduction in other forums is permitted, provided the original author(s) and the copyright owner(s) are credited and that the original publication in this journal is cited, in accordance with accepted academic practice. No use, distribution or reproduction is permitted which does not comply with these terms. 